\title{
Radio observations of planetary nebulae: no evidence for strong radial density gradients
}

DOI:

10.1093/mnras/sty 1673

\section{Document Version}

Accepted author manuscript

Link to publication record in Manchester Research Explorer

\section{Citation for published version (APA):}

Hajduk, M., Van Hoof, P. A. M., Sniadkowska, K., Krankowski, A., Blaszkiewicz, L., Dabrowski, B., \& Zijlstra, A. (2018). Radio observations of planetary nebulae: no evidence for strong radial density gradients. Royal Astronomical Society. Monthly Notices, 479(4). https://doi.org/10.1093/mnras/sty1673

\section{Published in:}

Royal Astronomical Society. Monthly Notices

\section{Citing this paper}

Please note that where the full-text provided on Manchester Research Explorer is the Author Accepted Manuscript or Proof version this may differ from the final Published version. If citing, it is advised that you check and use the publisher's definitive version.

\section{General rights}

Copyright and moral rights for the publications made accessible in the Research Explorer are retained by the authors and/or other copyright owners and it is a condition of accessing publications that users recognise and abide by the legal requirements associated with these rights.

\section{Takedown policy}

If you believe that this document breaches copyright please refer to the University of Manchester's Takedown Procedures [http://man.ac.uk/04Y6Bo] or contact uml.scholarlycommunications@manchester.ac.uk providing relevant details, so we can investigate your claim.

\section{OPEN ACCESS}




\title{
Radio observations of planetary nebulae: no evidence for strong radial density gradients
}

\author{
M. Hajduk, ${ }^{1 \star}$ P. A. M. van Hoof, ${ }^{2}$ K. Śniadkowska, ${ }^{1}$ A. Krankowski, ${ }^{1}$ \\ L. Błaszkiewicz, ${ }^{1,3}$ B. Dąbrowski, ${ }^{1}$ and A. A. Zijlstra ${ }^{4}$ \\ ${ }^{1}$ Space Radio-Diagnostics Research Centre, University of Warmia and Mazury in Olsztyn, Prawocheńskiego 9, 10-720 Olsztyn, Poland \\ ${ }^{2}$ Royal Observatory of Belgium, Ringlaan 3, B-1180 Brussels, Belgium \\ ${ }^{3}$ Faculty of Mathematics and Computer Sciences, University of Warmia and Mazury in Olsztyn, Stoneczna 54, 10-720 Olsztyn, Poland \\ ${ }^{4}$ Department of Astronomy and Astrophysics, The University of Manchester, Manchester, M13 9PL, UK
}

Accepted XXX. Received YYY; in original form ZZZ

\begin{abstract}
Radio continuum observations trace thermal emission of ionized plasma in planetary nebulae and bring useful information on nebular geometries. A model of homogeneous sphere or shell cannot fit the nebular spectra and brightness temperatures. Two alternative models have been proposed in the literature: the first one consists of two homogeneous components, while the other one is a model of a shell with a significant radial density gradient. On the other side, prolate ellipsoidal shell model can successfully fit the surface brightness distribution of selected objects. We verify the existing models using data collected in radio surveys covering wide range of frequencies. In about $50 \%$ cases, density gradient can be excluded, and none of the remaining objects could be confirmed. None of the observed planetary nebulae show the spectral index of 0.6 in the optically thick part of the spectrum, which is a value predicted for a shell containing strong radial density gradient. Radio spectra can be fitted with a model of prolate ellipsoidal shell, but also by a shell containing temperature variations in planetary nebulae. At least eight planetary nebulae show two component spectra, with one compact component showing much higher optical thickness than the other one. Unexpectedly, a group of planetary nebulae with lowest surface brightness show nonnegligible optical thickness. Their emission comes from compact and dense structures, comprising only small part of total nebular mass.
\end{abstract}

Key words: planetary nebulae: general - radio continuum: general

\section{INTRODUCTION}

Low and intermediate mass stars $\left(1-8 \mathrm{M}_{\odot}\right)$ play a vital role in the chemical evolution of the Galaxy (Marigo 2001). Up to $90 \%$ of their initial mass returns to the interstellar medium, most of it during the asymptotic giant branch (AGB) phase of their evolution.

After a long phase of steady hydrogen burning on the main sequence, hydrogen is exhausted in the center of the star and nuclear burning proceeds in a shell around a helium core. The star ascends the red giant branch in the Hertzsprung-Russell diagram. Subsequently, helium ignites in the core. After helium is exhausted in the core, helium burning proceeds in a shell on the top of carbon-oxygen core. The star ascends the asymptotic giant branch.

^ E-mail: marcin.hajduk@uwm.edu.pl
The main site of nucleosynthesis at the end of the AGB phase is the helium burning shell, separated from the hydrogen burning shell by the intershell region. Helium burning activates during the thermal pulse (helium flash), when enough helium is accumulated in quiescent hydrogen burning. The material synthesized during a thermal pulse is subsequently dredged up to the surface by the convective envelope.

Thermal pulses trigger heavy mass loss. When the envelope mass is reduced to $\sim 10^{-3} M_{\odot}$, the star begins to evolve very quickly to higher effective temperatures. Heavy mass loss terminates, and the slow and dense AGB wind is being compressed by a fast wind from the central star, which creates a central cavity. The density profile of the shell is further modified by the passage of the ionization front. A planetary nebula ( $\mathrm{PN}$, plural $\mathrm{PNe}$ ) becomes visible as a result of ionization and wind interaction. 


\section{RADIO EMISSION OF PLANETARY NEBULAE}

Planetary nebulae are detectable in a broad range of the electromagnetic spectrum, from UV or even $\mathrm{X}$ rays to radio frequencies. Continuum radio emission originates from thermal free-free emission of electrons (Pazderska et al. 2009; Chhetri et al. 2015). Radio observations trace all the ionized ejecta in the PN and give information on the nebular structure and physical parameters unaffected by extinction. This in turn can help to reveal the mass loss history of the star.

Spherically symmetric models cannot fit the observations. PNe show an excess of the $5 \mathrm{GHz} / 1.4 \mathrm{GHz}$ flux ratio with respect to a model of a homogeneous spherical shell. Siódmiak \& Tylenda (2001) introduced a model consisting of two components to fit the observations.

On the other hand, Phillips (2007) claimed that at least $10-20 \%$ of $\mathrm{PNe}$ are associated with strong density gradients, which can explain the observed $5 \mathrm{GHz} / 1.4 \mathrm{GHz}$ flux indices. For a shell with the density varying with radius $n_{e}(r) \propto r^{-2}$ the spectrum has a slope of $\sim 0.6$ in the range of frequencies.

Gruenwald \& Aleman (2007) were able to reproduce a range of spectral indices without introducing the initial density gradient, however, they analyzed the spectral indices limited to the frequency interval of 0.6 to $0.4 \mathrm{GHz}$.

The analysis using $5 \mathrm{GHz} / 1.4 \mathrm{GHz}$ flux ratio by Siódmiak \& Tylenda (2001) and Phillips (2007) did not lead to conclusive results. A prolate ellipsoidal shell was used by Masson (1990) and Aaquist \& Kwok (1996) to fit radio images of PNe. The prolate ellipsoidal shell, viewed at different angles, can reproduce variety of observed nebular morphologies.

With the advent of many radio surveys, more data is available for different frequencies in addition to $5 \mathrm{GHz}$ and $1.4 \mathrm{GHz}$ fluxes, widely used in previous studies. We take advantage of recently published catalogs of radio fluxes covering low and high frequency region to further constrain the proposed models. New data helped us to constrain the slope of radio spectra at optically thick part, which can verify the existence of strong density gradients. We also attempted to fit prolate ellipsoidal shell model and models with electron temperature varying across the nebula to the observed spectral indices.

\section{DATA}

We searched for archival radio observations of PNe having at least three observations at different frequencies in total, including at least one observation in low or high frequency surveys to ensure wide coverage in frequencies.

Low frequency surveys between 150 and $352 \mathrm{MHz}$ were carried on the Westerbork Synthesis Radio Telescope (Rengelink et al. 1997; Taylor et al. 1996; De Breuck et al. 2002), Giant Metrewave Radio Telescope (TGSS survey, Intema et al. 2017), Murchison Widefield Array (GLEAM survey, Hurley-Walker et al. 2017), and the Texas interferometer (Douglas et al. 1996). High frequency surveys were carried by Pazderska et al. (2009), Casassus et al. (2007), Umana et al. (2008), and the Planck Collaboration (2016). For intermediate frequencies we used data from surveys which derived fluxes with accuracy $\leq 10 \%$. The data are summarized in Table 1. We used SIMBAD database (Wenger et al. 2000) to identify spurious background sources.

We used nebular diameters from Frew et al. (2016). Radio diameters available in the literature become unreliable for small nebulae are less certain than optical diameters (Siódmiak \& Tylenda 2001). Frew et al. (2016) measured diameters for the emission region at the $10 \%$ level of the surface brightness, which should represent the shell contributing most of the flux in radio. In the case of non-spherical $\mathrm{PNe}$, we adopted the geometric mean of the two axes.

\section{MODELING OF RADIO SPECTRA}

For an isothermal nebula the radio flux $S_{v}$ is given by

$S_{v}=\frac{2 v^{2} k T_{e}}{c^{2}}\left(1-e^{-\tau_{v}}\right) \Omega$

and optical thickness of the nebula $\tau_{v}$

$\tau_{v}=5.44 \times 10^{-2} T_{e}^{-1.5} v^{-2} g_{f f}\left(v, T_{e}\right) \int n\left(H^{+}\right) n_{e} d S$

where $T_{e}$ is electron temperature, $\Omega$ is the solid angle, $n\left(H^{+}\right)$ and $n_{e}$ are proton and electron density, respectively. The Gaunt factor is given by $g_{f f}$. Emission measure $E M=$ $\int n_{e} n\left(H^{+}\right) d S$ is integrated along the line of sight. van Hoof et al. (2014) provided accurate values of the Gaunt factor $g_{f f}$.

We used five different models to fit observations: a homogeneous sphere, a cylindrical shell seen along its axis, a spherical shell with uniform density distribution, a spherical shell containing radial density gradient, and a prolate ellipsoidal shell. We also checked if $T_{e}$ variations in a $\mathrm{PN}$ could fit the observations.

\subsection{Model A: homogeneous sphere}

As a first approximation, we fitted a model of a homogeneous sphere. There are three free parameters in equations 1 and 2 : $\Omega, T_{e}$, and $E M$. EM primarily determines the turnover frequency $v$ of the PN spectrum for which $\tau_{v} \sim 1$. The optically thin part of the spectrum has a spectral index of about -0.1 . The absolute flux is proportional to $E M$. The optically thick part of the spectrum has a spectral index of 2 . The absolute flux is proportional to $T_{e}$. Additionally, $\Omega$ scales the absolute flux in the whole range of the spectrum. $E M$ and $T_{e}$, can be measured unambiguously when the radio spectrum covers both optically thick and thin emission. This is the case for most of PNe studied by us, which show decline of the flux due to increasing optical thickness at lowest frequencies.

Model A in most cases gave electron temperatures significantly lower than $\sim 10^{4} \mathrm{~K}$, expected for photo ionized nebula in equilibrium (Fig. 1). Such low temperatures contradict optical observations and physics of PNe. Higher $T_{e}$, however, would overestimate the flux in optically thick region in model A. Thus, model A is not relevant for most of PNe. 
Table 1. Radio continuum observations used for modeling of nebular spectra.

\begin{tabular}{|c|c|c|c|c|}
\hline frequency $[\mathrm{GHz}]$ & sky coverage & sensitivity $[\mathrm{mJy} / \mathrm{beam}]$ & beam size & reference \\
\hline 0.15 & $\delta>-56^{\circ}$ & 25 & $20^{\prime \prime}$ or $25^{\prime \prime} \times 25^{\prime \prime}$ & Intema et al. (2017) \\
\hline 0.2 & $\delta<+30^{\circ} \&|b|>10^{\circ}$ & 6.7 & see reference & Hurley-Walker et al. (2017) \\
\hline 0.325 & $\delta>+28^{\circ}$ & 30 & $54^{\prime \prime} \times 54^{\prime \prime} \operatorname{cosec} \delta$ & Rengelink et al. (1997) \\
\hline 0.330 & $+42<l<+92,|b|<1.6^{\circ}$ & 10 & $1^{\prime} \times 1^{\prime} \operatorname{cosec} \delta$ & Taylor et al. (1996) \\
\hline 0.352 & $-26^{\circ}<\delta<-9^{\circ}$ & 18 & $54^{\prime \prime} \times 54^{\prime \prime} \operatorname{cosec} \delta$ & De Breuck et al. (2002) \\
\hline 0.365 & $71.5^{\circ}>\delta>-35^{\circ}$ & 400 & see reference & Douglas et al. (1996) \\
\hline 0.843 & $\delta<-30^{\circ},|b|>10^{\circ}$ & $18(8$ for $\delta<-50)$ & $45^{\prime \prime} \times 45^{\prime \prime} \operatorname{cosec} \delta$ & Mauch et al. (2003) \\
\hline 0.843 & $|b|<-10^{\circ}, 245^{\circ}<l<365^{\circ}$ & $10(6$ for $\delta<-50)$ & $45^{\prime \prime} \times 45^{\prime \prime} \operatorname{cosec} \delta$ & Murphy et al. (2007) \\
\hline 1.4 & $\delta>-40^{\circ}$ & 2.5 & $45^{\prime \prime}$ & Condon et al. (1998) \\
\hline 1.4 & dedicated survey & see reference & $3.8^{\prime \prime} \times 5.5^{\prime \prime}$ & Isaacman (1984) \\
\hline 1.5 & dedicated survey & see reference & see reference & Zijlstra et al. (1989) \\
\hline 4.85 & $0^{\circ}<\delta<75^{\circ}$ & 25 & $3.6^{\prime} \times 3.4^{\prime}$ & Gregory et al. (1996) \\
\hline 4.85 & $-87.5^{\circ}<\delta<10^{\circ}$ & 35 & $4.2^{\prime}$ & Griffith \& Wright (1993) \\
\hline 4.85 & dedicated survey & see reference & $2.8^{\prime \prime} \times 1.5^{\prime \prime}$ & McConnell (2012) \\
\hline 5 & dedicated survey & see reference & $4.9^{\prime \prime} \times 6.5^{\prime \prime}$ & Isaacman (1984) \\
\hline 5 & dedicated survey & see reference & see reference & Zijlstra et al. (1989) \\
\hline 5 & dedicated survey & see reference & $0.5^{\prime \prime}$ & Aaquist \& Kwok (1990) \\
\hline 5 & $\delta<27^{\circ}$ & see reference & $4.5^{\prime \prime}$ & Milne \& Aller (1975) \\
\hline 5 & $\delta<0^{\circ}$ & 40 & see reference & Murphy et al. (2010) \\
\hline 8 & $\delta<0^{\circ}$ & 40 & see reference & Murphy et al. (2010) \\
\hline 8.6 & dedicated survey & see reference & $1.3^{\prime \prime} \times 0.9^{\prime \prime}$ & McConnell (2012) \\
\hline 14.7 & $\delta<27^{\circ}$ & see reference & $2.1^{\prime \prime}$ & Milne \& Aller (1982) \\
\hline 15 & dedicated survey & see reference & see reference & Zijlstra et al. (1989) \\
\hline 15 & dedicated survey & see reference & $0.1^{\prime \prime}$ & Aaquist \& Kwok (1996) \\
\hline 16 & $|b| \leq 5,170^{\circ}>l>76^{\circ}$ & 16 & $3^{\prime}$ & Perrott et al. (2015) \\
\hline 20 & $\delta<0^{\circ}$ & 40 & see reference & Murphy et al. (2010) \\
\hline 30 & $\delta>-15^{\circ}$ & 20 & $1.2^{\prime}$ & Pazderska et al. (2009) \\
\hline 30 & all sky & 427 & $32^{\prime}$ & Planck Collaboration (2016) \\
\hline 31 & dedicated survey & 20 & $45.2^{\prime \prime}$ & Casassus et al. (2007) \\
\hline 43 & dedicated survey & 50 & $54^{\prime \prime}$ & Umana et al. (2008) \\
\hline 44 & all sky & 692 & $27^{\prime}$ & Planck Collaboration (2016) \\
\hline 70 & all sky & 501 & $13.2^{\prime}$ & Planck Collaboration (2016) \\
\hline 100 & all sky & 269 & $9.7^{\prime}$ & Planck Collaboration (2016) \\
\hline 143 & all sky & 177 & $7.2^{\prime}$ & Planck Collaboration (2016) \\
\hline 217 & all sky & 152 & $4.9^{\prime}$ & Planck Collaboration (2016) \\
\hline 353 & all sky & 304 & $4.9^{\prime}$ & Planck Collaboration (2016) \\
\hline 353 & dedicated survey & 71 & $14^{\prime \prime}$ & Di Francesco et al. (2008) \\
\hline
\end{tabular}

\subsection{Model B: homogeneous shell}

In order to derive more physically plausible models, we fixed $T_{e}$ to the value derived from optical spectra $T_{o p t}$ (Cahn et al. 1992). We used a model of uniform sphere, but with a central cavity defined by the ratio of the inner to outer radius $\eta_{B}=R_{i n} / R$. The fitted parameters in model $\mathrm{B}$ were $\eta_{B}$ and $E M$.

The shell model with $\eta_{B} \approx 1$ shows strong limb brightening. The $E M$ reaches the highest value at the projected distance of $R_{i n}$ from the center of the PN and is low elsewhere, which may reproduce the observations. Model B with $\eta_{B} \approx 1$ would require a very high density and would produce ring shaped $\mathrm{PNe}$. On the other side, model $\mathrm{B}$ with $\eta_{B} \approx 0$ is similar to model A.

The fitted $E M$ reproduced well the flux density but was too low to reproduce the turnover frequency in many cases (Fig. 1). Higher $E M$ would fit the turnover frequency better, but also would overestimate the absolute flux in optically thin part of the spectrum. Thus, model B is not relevant for most of PNe.

\subsection{Model C: homogeneous cylindrical shell}

We introduced the $\left(1-\eta^{2}\right)$ factor in equation 1 so that we could increase $E M$ to achieve a good fit of the turnover region and simultaneously scale down the absolute flux:

$S_{v}=\frac{2 v^{2} k T_{e}}{c^{2}}\left(1-e^{-\tau_{v}}\right) \Omega\left(1-\eta^{2}\right)$

where fitted parameters are $\eta$ and $E M$. The $\eta$ parameter is defined as the ratio of the projected inner to outer radius $R_{\text {in }} / R$.

Model $\mathrm{C}$ reproduced most of the spectra very well. It can be interpreted as a cylindrical shell seen along its axis, with the ratio of the internal to external radii defined by $\eta$. Model $\mathrm{C}$ can be relevant as a first approximation for some PNe, including the well known NGC 6720, which shows a disk seen pole on (O'Dell et al. 2013). Assuming random orientation of $\mathrm{PNe}$ in space, this model cannot account for most of PNe.

However, we find this model useful, as it is sensitive to the most optically thick part of the PN. The $\eta$ parameter gives information on surface brightness distribution of a PN. 

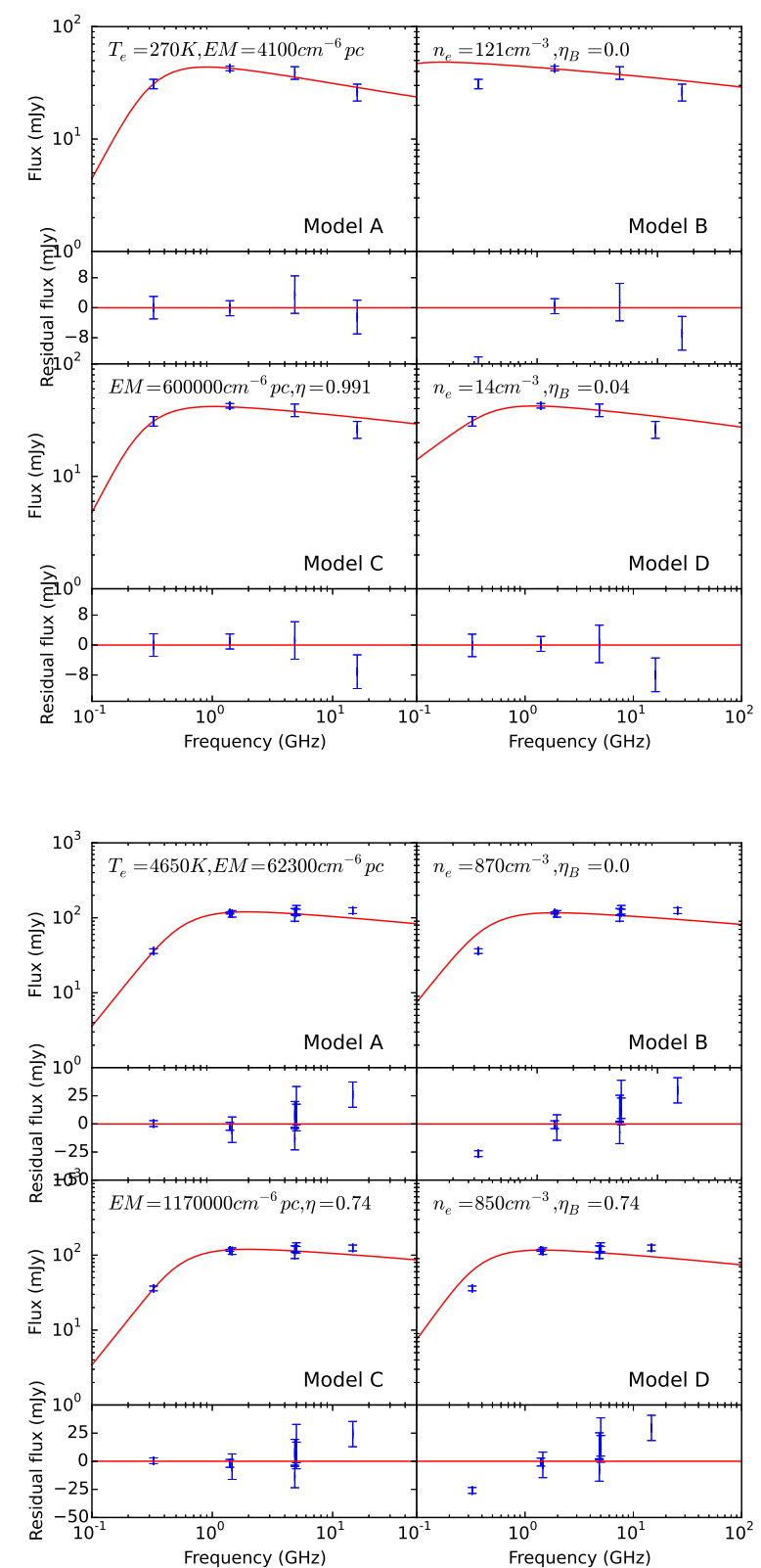

Figure 1. Four fits to the radio spectra of PN NGC 6842 (top) and IC 4634 (bottom) using models A, B, C, and D. The derived parameters for each model are shown. Lower panels show the residuals of the fits.

PNe with $\eta \approx 0$ can be fit equally well using model A. Almost all PNe require $\eta>0$ (Fig. 6). Low surface brightness PNe need $\eta$ very close to 1 . High value of $\eta$ indicates, that a small region with high $E M$ dominates in radio.

Fig. 2 presents the relation between emission measure derived in model $\mathrm{C}$ and dereddened $\mathrm{H} \alpha$ surface brightness (Frew et al. 2016). As expected, EM decreases with $S_{0}(\mathrm{H} \alpha)$, but unexpectedly this tendency is not held for PNe with the lowest surface brightnesses.

We also used a model consisting of two components, fitting two different values of $E M$ and $\eta$. In eight cases two

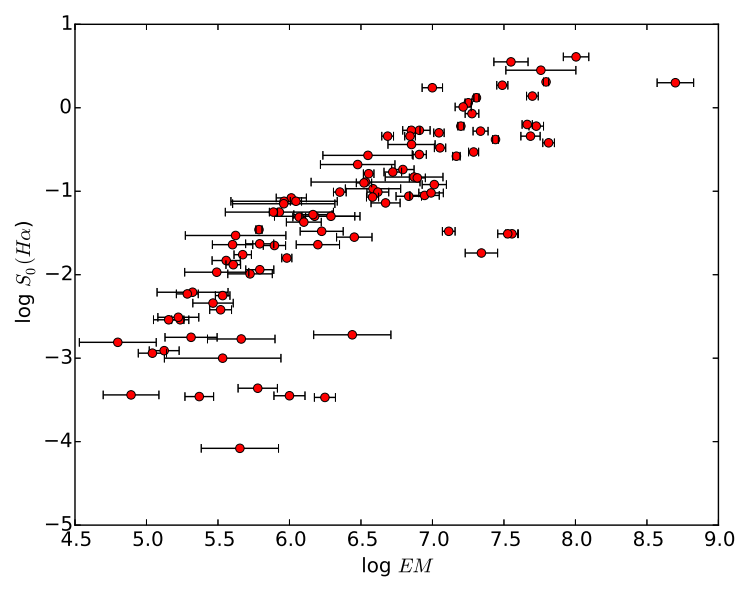

Figure 2. Emission measure vs $\mathrm{H} \alpha$ surface brightness in observed PNe.

components improves the fit significantly (Fig. 3). One of the components has much higher turnover frequency and a flux comparable to the second component. This indicates much higher $E M$ of the order of $10^{8} \mathrm{~cm}^{-6} p c$, which could indicate a compact and dense (possibly cool) component in a PN.

In the appendix we list $E M$ and $\eta$ fitted to equation 3 . In addition, we present optical depth at $5 \mathrm{GHz}$, brightness temperature at $5 \mathrm{GHz}$ derived from fitting the spectrum $T_{b}^{\text {spect }}=T_{e} \times\left(1-e^{-\tau_{5 G H z}}\right)$, and from $T_{b}^{\text {diam }}=73.87 F_{5 G H z} / \Theta^{2}$, where $\Theta$ is the diameter of the PN measured in arcsec, and $F_{5 \mathrm{GHz}}$ is flux at $5 \mathrm{GHz}$ measured in mJy.

$T_{b}^{\text {spect }}$ is systemically higher than $T_{b}^{\text {diam }}$ (Fig. 4). For uniform brightness distribution, corresponding to $\eta=0$, $T_{b}^{\text {spect }}=T_{B}^{\text {diam }}$. For non uniform brightness distribution, $T_{b}^{\text {spect }}>T_{B}^{\text {diam }}$. The ratio of $T_{b}^{\text {spect }} / T_{B}^{\text {diam }}$ in Model C simply depends on $\eta: T_{b}^{\text {spect }} / T_{B}^{\text {diam }}=1-\eta^{2}$.

We derived ionized masses using distances derived by Frew et al. (2016) and emission measures from radio spectra using model C. Ionized masses increase with decreasing surface brightness down to $\log \left(S_{O}(\mathrm{H} \alpha)\right)=-2$ (Fig. 5) which reflects the progressing ionization in the nebulae (Frew et al. 2016). For lower surface brightness most of the observed PNe have $\eta>0.9$. Fig. 5 includes only $\mathrm{PNe}$ with reliable determination of $E M$, so low surface brightness $\mathrm{PNe}$ which are optically thin in radio are not included.

\subsection{Model D: a shell with a density gradient $\beta=2$}

We also used the modified model of power-law distribution by Olnon (1975) for $n_{e}=n_{o}\left(r /\left(\eta_{B} \times R\right)\right)^{-\beta}$ for $\eta_{B} \times R>r>R$, $n=0$ for $r \leq \eta_{B} \times R$, and $\beta=2$. This density distribution can result from a steady-state wind lasting for a finite time interval. In this model we fitted $\eta$ and $n_{o}$.

Model D results in the flat spectrum in an optically thin part and in a spectral index of 0.6 in partially optically thick part, and an index of 2 in the optically thick part. The range of spectrum where spectral index is 0.6 depends on the value of $\eta_{B}$ : the lower $\eta_{B}$, the larger range of frequencies where the spectral index of 0.6 is observed. 

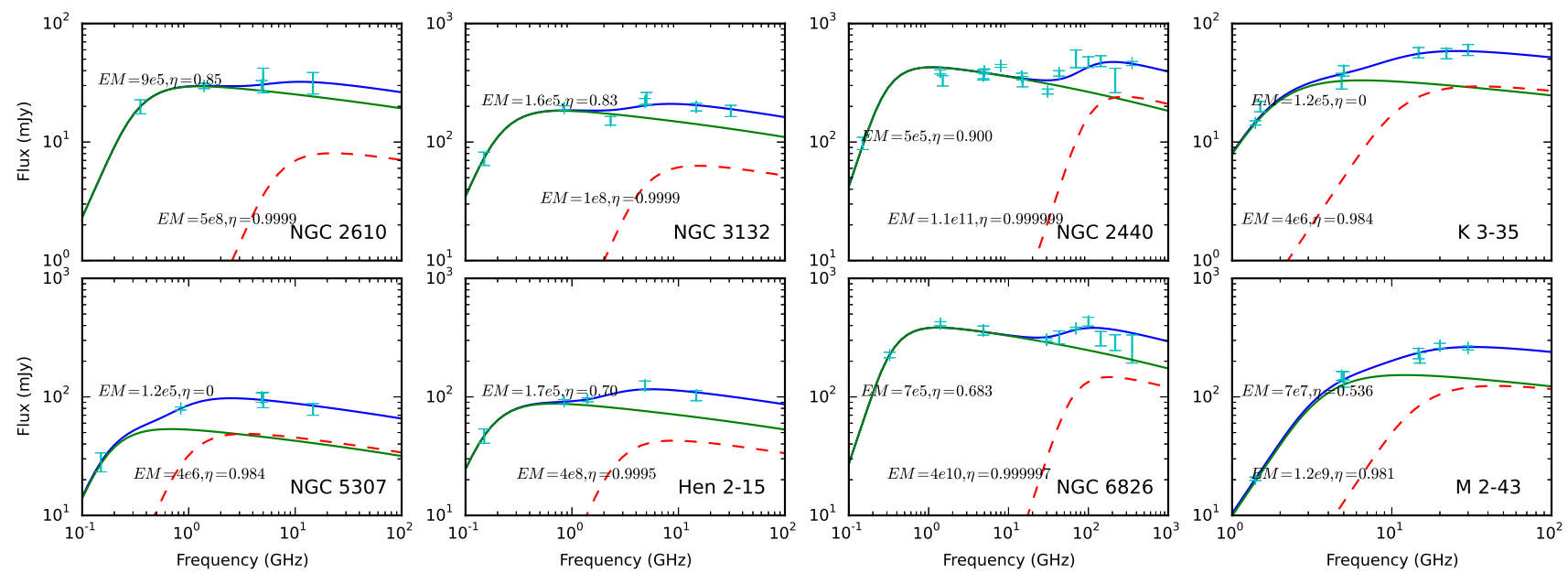

Figure 3. PNe showing two components in their spectra. Dashed red and green lines show separate components, blue line shows the sum of both components.

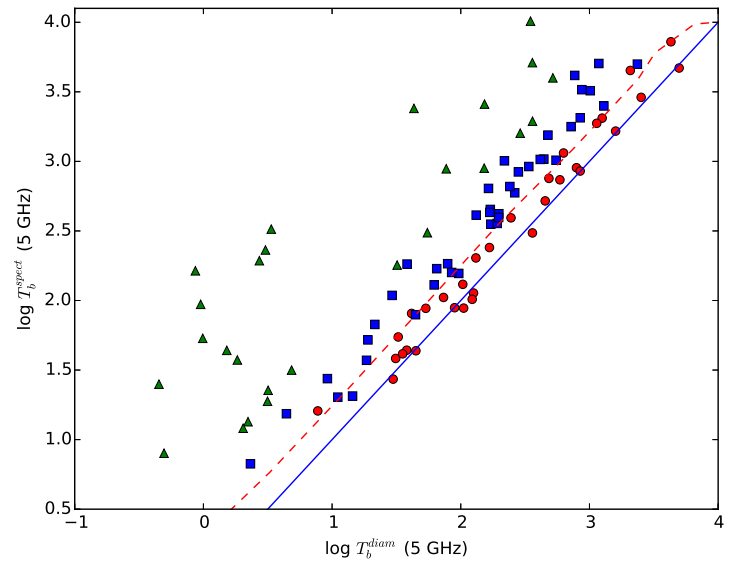

Figure 4. Brightness temperatures determined from measured diameter and flux and from radio spectra. PNe are split into three groups: $\eta<0.5$ (red circles), $0.5<\eta<0.9$ (blue squares), and $\eta>0.9$ (green triangles). Dashed red curve shows Model E for $a / b=3, t / b=0.1$, and $i=0^{\circ}$, and blue solid curve shows $T_{b}^{\text {diam }}=$ $T_{b}^{\text {spec }}$ relation.

\subsection{Model E: prolate ellipsoidal shell}

Surface radio brightness distribution suggests that a model of a prolate ellipsoidal shell can be representative for most of PNe (Masson 1990; Aaquist \& Kwok 1996). The inner and outer shell are defined as ellipsoids with minor and major axes of $(a, b)$ and $(a+t, b+t)$. Electron density depends on the incident flux, so that $n_{e}^{2} \times t$ is inversely proportional to the inner radius.

Due to large parameter space (inclinaton angle $i, a / b$ ratio, $t, n_{e}$ ), we did not use Model $\mathrm{E}$ to fit individual $\mathrm{PNe}$, but to fit spectral indices and brightness temperatures.

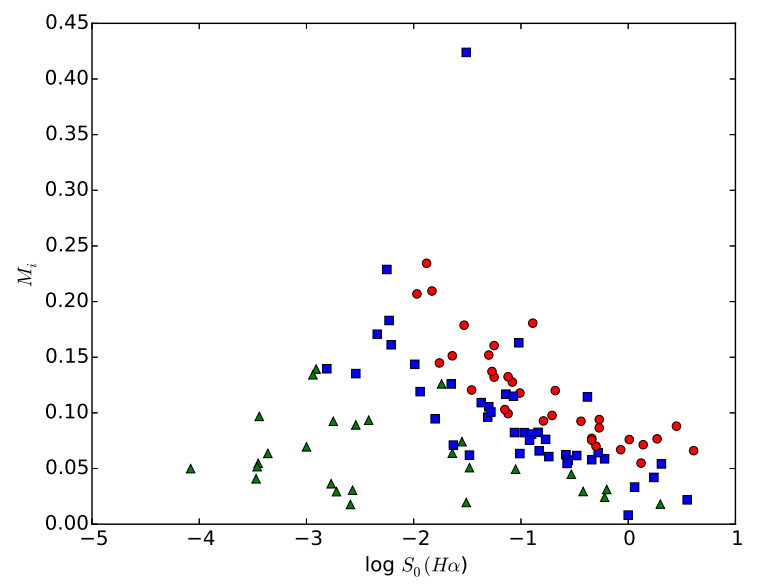

Figure 5. Ionized mass vs dereddened $H \boldsymbol{\alpha}$ surface brightness.

\subsection{Model F: temperature stratification}

We also explored the influence of temperature stratification on the radio spectra of $\mathrm{PNe}$. We assumed a spherical model with $T_{e}$ of $2500 \mathrm{~K}$ in the central region and $10000 \mathrm{~K}$ elsewhere, and a model with $T_{e}$ of $1000 \mathrm{~K}$ in the outer $20 \%$ radius and $10000 \mathrm{~K}$ inside. The temperature stratification is set arbitrarily. Again, we did not use this model for individual PNe, but rather to fit all PNe in the brightness temperature - spectral index diagrams.

\section{DISCUSSION}

\subsection{Spectral indices}

A diagram of spectral indices vs $T_{b}$ reveal an excess of the observed flux ratios with respect to model A and B (Fig. 7, $8)$. The excess does not depend on the nebular diameter nor on the instrument used (Fig. 9), except for Milne \& Aller 


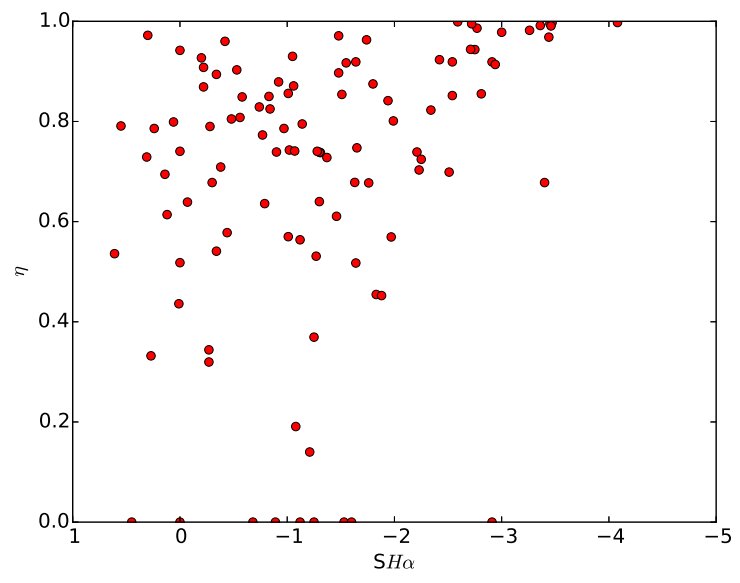

Figure 6. Diagram showing $\eta$ vs $\mathrm{H} \alpha$ surface brightness.

(1975), where the fluxes appear to be correlated with nebular size. Data by Milne \& Aller (1982) at higher frequencies show a similar problem. The data published by Perrott et al. (2015) appear to be underestimated. Apart from that the different instruments used does not seem to affect the observed indices.

In some cases, the excess is visible only at $5 \mathrm{GHz}$, e.g. in IC 3568, IC 5217, IC 289, or NGC 6072. Out of them, only IC 5217 shows a weak, confusing source within the primary beam of the survey of 3.5 arcmin (Gregory et al. 1996), which might be responsible for the excess of the $5 \mathrm{GHz} / 1.4 \mathrm{GHz}$ ratio. However, the excess of the $5 \mathrm{GHz} / 1.4 \mathrm{GHz}$ ratio does not depend on the PNe diameters, and thus cannot be affected by the presence of confusing sources in the single dish surveys. Also, $5 \mathrm{GHz}$ excess was reported by Siódmiak \& Tylenda (2001), who used only interferometric fluxes.

\subsection{Flux evolution}

One possible explanation for the $5 \mathrm{GHz}$ excess could be the evolution of the radio fluxes due to nebular expansion and change of the ionizing flux from the central star. $5 \mathrm{GHz}$ fluxes used in our paper are predominantly from the single dish surveys (Gregory et al. 1996; Griffith \& Wright 1993) carried out in the period 1986-1987 and 1990. 1.4 GHz observations were taken predominately from the NRAO VLA Sky Survey (NVSS) between 1993 and 1996. Since $1.4 \mathrm{GHz}$ flux should increase with respect to the $5 \mathrm{GHz}$ thorough the PN evolution, the time gap between the $5 \mathrm{GHz}$ surveys and NVSS should rather result in lowering the $5 \mathrm{GHz} / 1.4 \mathrm{GHz}$ ratio.

Zijlstra et al. (1989) reported variability of radio flux of NGC 7027 due to expansion of the nebula (increase of $0.25 \% y r^{-1}$ ) and decreasing number of ionizing photons (change of $-0.14 \% y^{-1}$ ). Such small changes would be difficult to detect with the precision of about $10 \%$ and relatively short time span between observations. However, flux evolution depends on the age of the nebula and pace of evolution of central star.

We checked the possible flux evolution using observations at the same frequency made for the same PN in different epochs, if available. We selected sources which showed
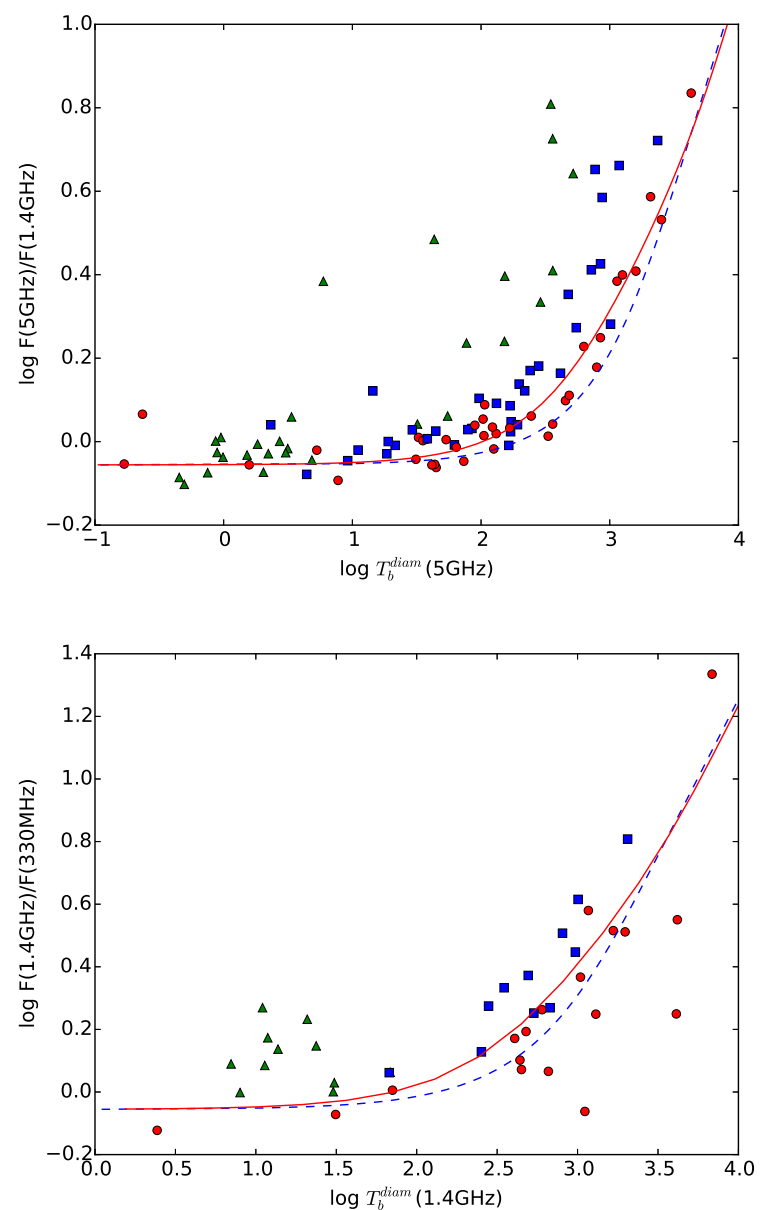

Figure 7. The observed spectral indices vs brightness temperature for PNe and model A for $T_{e}=10000 \mathrm{~K}$ (blue line). Red curves show Model E for $a / b=3, t / b=0.1$, and $i=0^{\circ}$. PNe are marked with the symbols as in Fig. 4.

more than $3 \sigma$ deviation in measurements at the same frequency. In most cases, the flux measurements did not show significant changes. We rejected sources in which the possible reason for discrepancy was systemic flux error at one epoch, which did not show the expected flux changes at other frequencies and the overall flux spectrum did not show any signatures of variability, e.g. in $\mathrm{Hu} 1-2$.

The variability of radio flux in IC 4997 has already been studied by Miranda \& Torrelles (1998). They showed yearto-year changes of the morphology of PN IC 4997 in radio due to interaction of the collimated stellar wind with the outer shell. In the archival data, the flux at optically thin part dropped from $100 \mathrm{mJy}$ around 1980 down to $50 \mathrm{mJy}$ about 2000. More recent data at higher frequencies show possible increase from 80 to 110 mJy between $2001-2002$ (Casassus et al. 2007) and 2005 - 2007 (Pazderska et al. 2009), respectively. However, the data are too sparse to fit the spectrum for each epoch separately.

Another case is M 2-2. This is a relatively compact PN with the diameter of only 6 arcsec. Zijlstra et al. (1989) observed higher values at $5 \mathrm{GHz}$ and $15 \mathrm{GHz}$, and lower flux at $1.5 \mathrm{GHz}$ than more recent observations. This would im- 

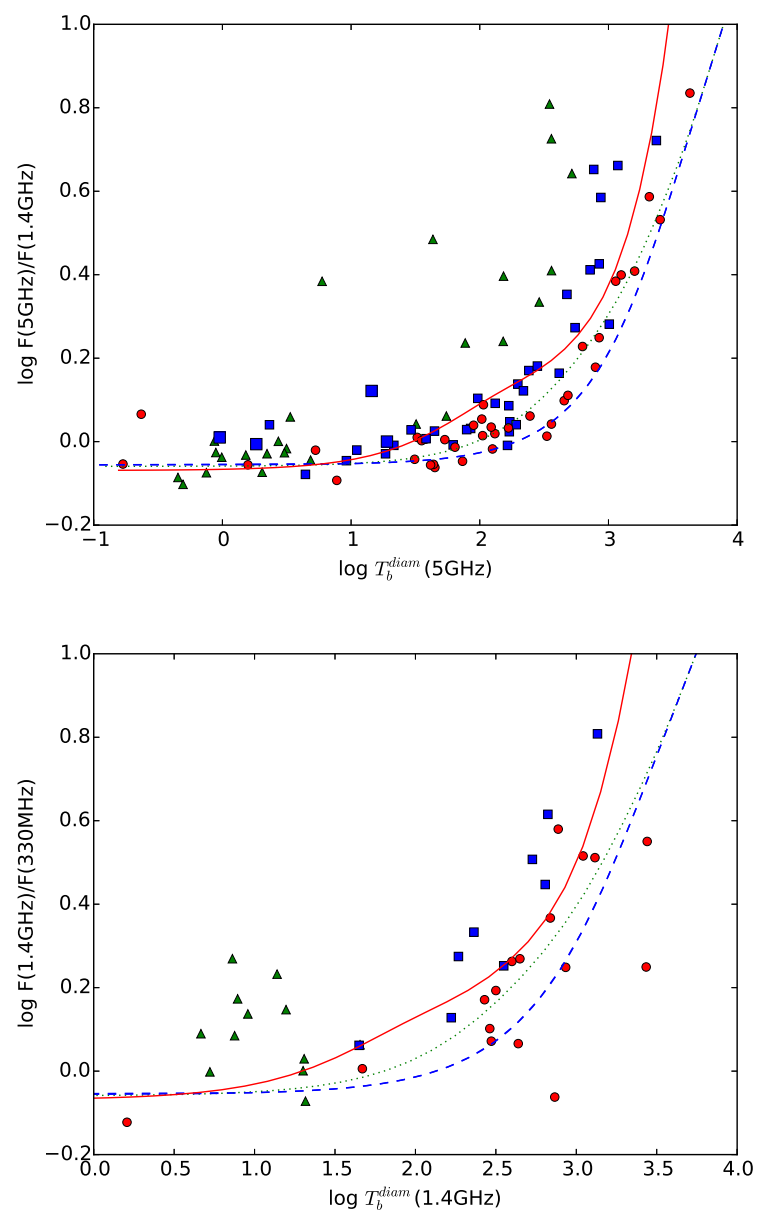

Figure 8. The observed spectral indices vs brightness temperature for $\mathrm{PNe}$ and model A for $T_{e}=10000 \mathrm{~K}$ (blue dashed line). Dotted curve shows model with inner region of lower temperature, while red solid curve shows model with outer region of lower temperature. PNe are marked with the symbols as in Fig. 4.

ply the lowering flux in continuum and increasing flux in optically thick part of the spectrum, in qualitative agreement with NGC 7027 (Zijlstra et al. 1989). Another possible variable is $\mathrm{M} 1-40$, showing a decreasing trend over time. We would need more data to analyze the flux variability in more details. Most of PNe did not show any variability.

\subsection{Radial density gradient}

A spectral index of 0.6 is an indication of a strong radial density gradient $(\beta=2)$. The spectral index of 0.6 should be relatively easy to confirm due to a relatively high flux at low frequencies. Phillips (2007) and Phillips (2007a) list candidates for strong density gradient. Eight of them are in common with our sample (Fig. 10). The objects were well fitted using model $\mathrm{C}$ except for Hu 1-2. However, the diameter of $\mathrm{Hu}$ 1-2 reported by Frew et al. (2016) apparently refers to the brightest, central region of the $\mathrm{PN}$, while apparently much larger region of the nebula contributes to the observed radio flux (Fang et al. 2015). With larger diameter we received much better fit.
Most of PNe could still be fitted with model D, but it yields worse or unrealistic fit compared to model $\mathrm{C}$ in about $50 \%$ of PNe. For a small value of $\eta_{B}$ model B gives artificially small densities $n_{o}$ or cannot fit the slope steeper than 0.6 in the optically thick part of the spectrum (e.g. Fig. 1, 10). Small $\eta_{B}$ would also result in the surface brightness quickly fading with the distance from the center of $\mathrm{PN}$, and this is not confirmed in radio images (Aaquist \& Kwok 1996).

Alternatively, the observed $\mathrm{PNe}$ may only have thin shells. Then, the spectral index of 0.6 is observed only in a very limited range of frequencies and is difficult to confirm. However, Model D is almost equivalent to model B for large $\eta_{B}$, and cannot fit the observations of most of PNe for the same reason, described in Section 4.2.

\subsection{Prolate ellipsoidal shell and temperature variations}

Model E explains well the observed spectral indices of many $\mathrm{PNe}$ with a typical value of $\mathrm{a} / \mathrm{b}$ of about 3 . Alternatively, observed spectral indices could result from temperature variations. Lower temperature in the outer layer results also in lower spectral index with respect to a homogeneous model in the optically thick part of the spectrum. It would eventually produce a spectral index steeper than 2 .

An upper limit from the TGSS survey indicates that some PNe show steeper spectral index than 2 in the optically thick part: NGC 6826, NGC 6543, and NGC 6818. Optically thick radio emission represents the emitting region at $\tau \sim 2 / 3$, whereas optically thin emission depends on volume averaged electron temperature. Thus, at $5 \mathrm{GHz}$ the excess can be interpreted as the temperature drop in the outermost regions of PN. The drop of temperature in the outer regions is not confirmed by spatially resolved spectroscopy of PNe (Sandin et al. 2008).

Model $\mathrm{F}$ with the temperature of $1000 \mathrm{~K}$ in the central region of the shell gives more flux in the optically thin region, as the emissivity of lower temperature region is higher. The optically thick spectrum is the same for the two models as radio emission originates from the outermost region of the shell. The region with lower temperature was indicated by Tsamis et al. (2004) and is one of the alternative explanations for the abundance discrepancy problem in $\mathrm{PNe}$. García-Rojas et al. (2017) showed that emission originating from optical recombination lines is more centrally concentrated than emission from collisionally excited lines.

\subsection{Low surface brightness $\mathrm{PNe}$}

Unexpectedly, a group of PNe with low $T_{b}^{\text {diam }}$ shows nonnegligible optical thickness at lowest frequencies (Figs. 7,8). Model $\mathrm{C}$ gives $\eta>0.9$, which indicates that a small part of the projected area of the PN with high $E M$ contribute most of the radio flux. However, NVSS and other catalogs give the radii comparable to that seen in optical. This suggests that they form a cylindrical shell observed along its axis, which causes strong limb brightening, or contain a compact component, which contribution in flux is comparable to the rest of PN. Relatively low $M_{i}$ indicate that these structures comprise only part of the nebular ionized mass.

Figure 6 shows, that for low surface brightness PNe 

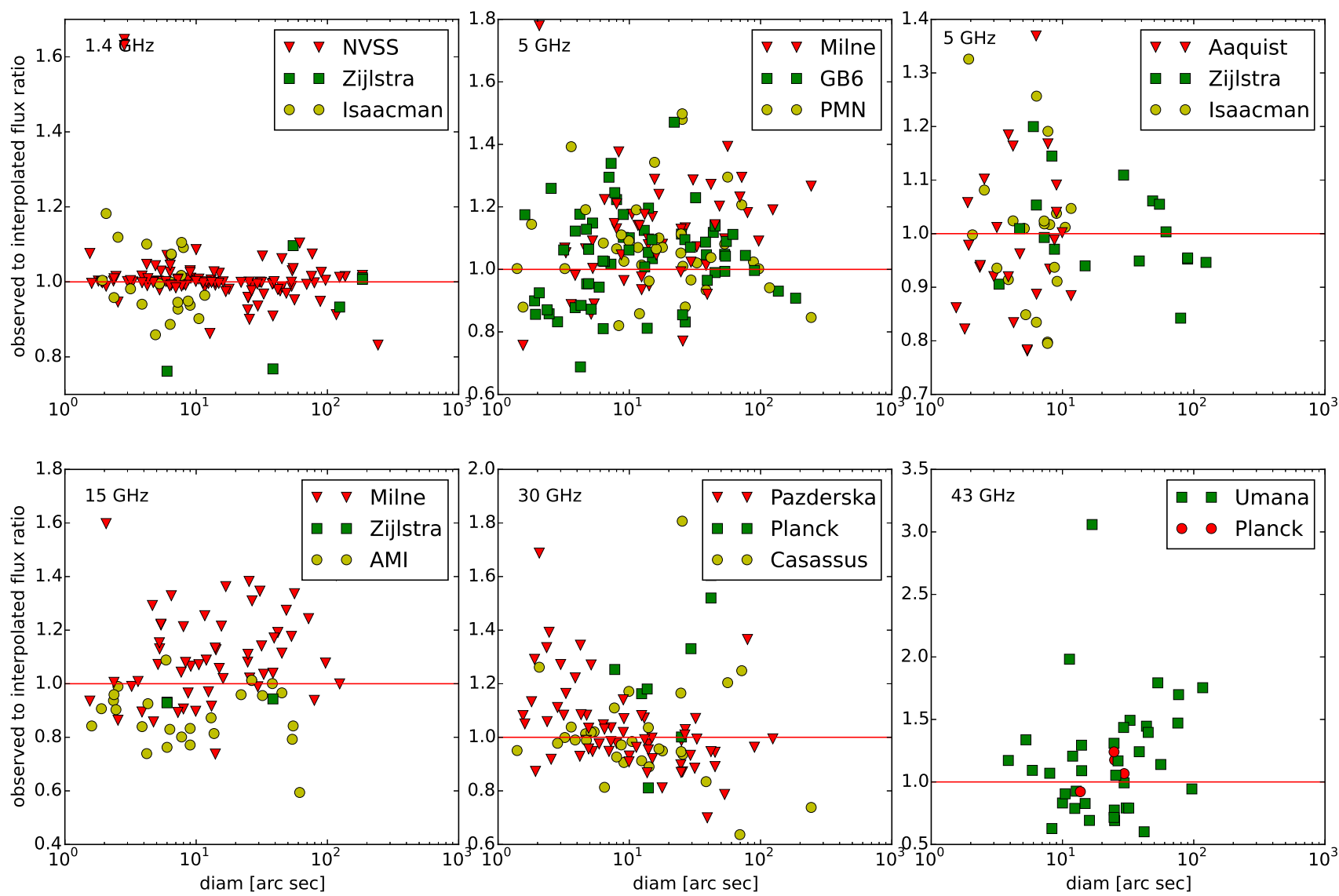

Figure 9. The ratio of the observed flux to the flux derived for model C vs PN diameter for different frequencies. For $5 \mathrm{GHz}$, single dish and interferometric surveys are shown separately.
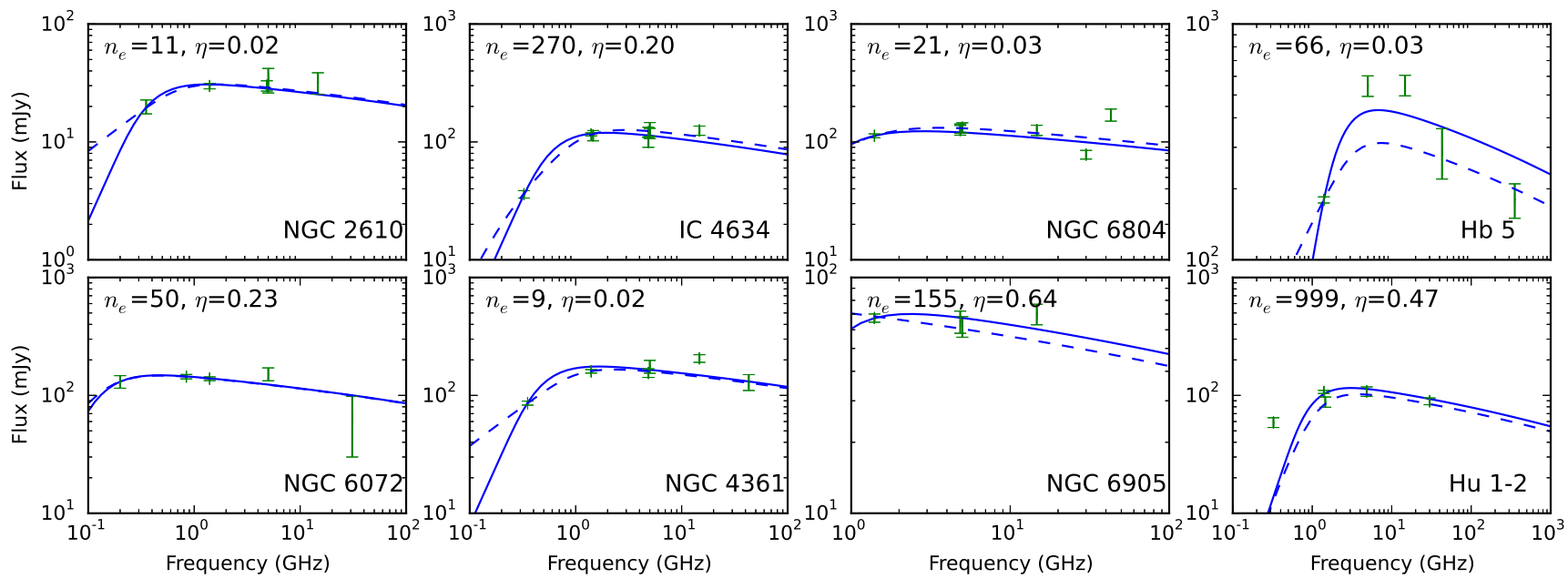

Figure 10. Spectra of PNe claimed to have strong density gradient. Solid line shows fit of model C with the parameters given in the appendix, and dashed line shows fit of model D with the parameters indicated. 
$S_{0}(\mathrm{H} \alpha)<-2$ only those with large $\eta$ parameter are observed. According to Aaquist \& Kwok (1996), morphology of PNe does not change between low and high surface brightness $\mathrm{PNe}$. This indicates, that low surface brightness $\mathrm{PNe}$ are detected only when they contain a small region with high column density.

This group contains bipolar or quadrupolar $\mathrm{PNe}$ NGC 650-51, NGC 4361, NGC 6302, or M 1-75. Also, three PNe classified as round, NGC 2610, Abell 53, and NGC 6842, belong to this group, indicating, that their spatial structure is more complex than spherical.

\section{SUMMARY}

In conclusion, we confirm that homogeneous models cannot fit the observed radio spectra and brightness temperatures of most of PNe. We do not find an evidence for a strong radial density gradient in $\mathrm{PNe}$. We found a sample of $\mathrm{PNe}$ showing two component spectra, but they cannot account for the observed $5 \mathrm{GHz}$ to $1.4 \mathrm{GHz}$ indices. A model of prolate ellipsoidal shell or temperature variations explain very well the observed spectral indices with except of low surface brightness PNe. Part of low surface brightness PNe are only observed in radio due to their specific morphology. Further observations at low frequencies having better sensitivity and resolution, e.g. Low Frequency Array (van Haarlem et al. 2013) will better constrain the spectral index in optically thick part of the spectra and geometry of PNe.

\section{ACKNOWLEDGEMENTS}

We thank to the Ministry of Science and Higher Education (MSHE) of Republic of Poland for granting funds for Polish contribution to the International LOFAR Telescope (MSHE decision no DIR/WK//2016/05) and for maintenance of the LOFAR PL- 612 Baldy (MSHE decision no 220815/E-383/SPUB/2016/2). We gratefully acknowledge financial support from National Science Centre, Poland, grant No. 2016/23/B/ST9/01653. This research has made use of the SIMBAD database, operated at CDS, Strasbourg, France.

\section{REFERENCES}

Acker, A., Marcout, J., Ochsenbein, F., et al. 1992, The Strasbourg-ESO Catalogue of Galactic Planetary Nebulae, Parts I, II

Aaquist, O. B. \& Kwok, S. 1990, A\&AS, 84, 229

Aaquist, O. B. \& Kwok, S. 1996, ApJ, 462, 813

Cahn, J. H., Kaler, J. B. \& Stanghellini, L. 1992, A\&AS, 94, 399

Casassus, S., Nyman, L.- $\varnothing$ A., Dickinson, C., Pearson, T. J. 2007, MNRAS, 382, 1607

Chhetri, R., Ekers, R. D., Kimball, A., et al. 2007, MNRAS, 382, 1607

Condon, J. J., Cotton, W. D., Greisen, E. W., et al. 1998, AJ, 115,1693

De Breuck, C., Tang, Y., de Bruyn, A. G. Röttgering, H. \& van Breugel, W. 2002, A\&A, 394, 59

Di Francesco, J., Johnstone, D., Kirk, H., MacKenzie, T. \& Ledwosinska, E. 2008, ApJS, 175, 277
Douglas, J. N., Bash, F. N., Bozyan, F. A., Torrence, G. W. \& Wolfe, C. 1996, AJ, 111, 1945

Fang, X., Guerrero, M. A., Miranda, L. F., et al. 2015, MNRAS, 452,2445

Frew, D. J., Parker, Q. A., Bojičić, I. S. 2016, MNRAS, 455, 1459

García-Rojas, J., et al. 2017, IAUS, 323, 65

Gregory, P. C., Scott, W. K., Douglas, K. \& Condon, J. J. 1996, ApJS, 103, 427

Griffith, M. R., Wright, A. E. 1993, AJ, 105, 1666

Gruenwald, R., Aleman, A. 2007, A\&A, 461, 1019

Hurley-Walker, N., Callingham, J. R., Hancock, P. J., et al. 2017, MNRAS, 464, 1146

Intema, H. T., Jagannathan, P., Mooley, K. P. \& Frail, D. A. 2017, A\&A, 598A, 78

Isaacman, R. 1984, MNRAS, 208, 399

Kerber, F., Mignani, R. P., Guglielmetti, F. \& Wicenec, A. 2003, A\&A, 408, 1029

Marigo, P. 2001, A\&A, 370, 194

Masson, C. R. 1990, ApJ, 348, 580

Mauch, T., Murphy, T., Buttery, H. J., et al. 2003, MNRAS, 342, 1117

McConnell, D., Sadler, E. M., Murphy, T., Ekers, R. D. 2012, MNRAS, 422, 1527

Milne, D. K. \& Aller, L. H. 1975, A\&A, 38, 183

Milne, D. K. \& Aller, L. H. 1982, A\&AS, 50, 209

Miranda, L. F.; Torrelles, J. M. 1998, ApJ, 496, 274

Monteiro, H., Morisset, C., Gruenwald, R., Viegas, S. M. 2000, ApJ, 537, 853

Murphy, T., Mauch, T., Green, A. et al. 2007, MNRAS, 382, 382

Murphy, T., Sadler, E. M., Ekers, R. D. et al. 2010, MNRAS, 402, 2403

O'Dell, C. R., Ferland, G. J., Henney, W. J., Peimbert, M. 2013, AJ, 145, $170 \mathrm{O}$

Olnon, F. M. 1975, A\&A, 39, 217

Pazderska, B. M., Gawroński, M. P., Feiler, R., et al. 2009, A\&A, 498,463

Perrott, Y. C., Scaife, A. M. M., Green, D. A., et al. 2015, MNRAS, 453, 1396

Phillips, J. P. 2007, MNRAS, 378, 231

Phillips, J. P. 2007, RMxAA, 43, 303

Planck Collaboration XXVI. 2016, A\&A, 594, A26

Rengelink, R. B., Tang, Y., de Bruyn, A. G., et al. 1997, A\&AS, 124,259

Sahai, R., Morris, M. R., Villar, G. G. 2011, AJ, 141, 134S

Sandin, R., et al. 2008, A\&A, 486, 545

Siódmiak, N., Tylenda, R. 2001, A\&A, 373, 1032

Taylor, A. R., Goss, W. M., Coleman, P. H., van Leeuwen, J. \& Wallace, B. J. 1996, ApJS, 107, 239

Taylor, A. R., Pottasch, S. R., Zhang, C. Y. 1987, A\&A, 171, 178

Tsamis, Y. G., Barlow, M. J., Liu, X. W., Storey, P. J., Danziger, I. J. 2004, MNRAS, 353, 953

Umana, G., Leto, P., Trigilio, C., et al. 2008, A\&A, 482, 529

van Haarlem, M. P., Wise, M. W., Gunst, A. W., et al. 2013, A\&A, 556A, 2

van Hoof, P. A. M., Williams, R. J. R., Volk, K., et al. 2014, MNRAS, 444, 420

Wenger, M., Ochsenbein, F., Egret, D., et al. 2000, A\&AS, 143, $9 \mathrm{~W}$

Zijlstra, A. A., Pottasch, S. R., Bignell, C., et al. 1989, A\&AS, 79,329

Zijlstra, A. A., van Hoof, P. A. M., Perley, R. A. 2008, ApJ, 681, 1296

APPENDIX A: EMISSION MEASURES AND $\eta$ VALUES DERIVED FOR MODEL C 
Table A1: Modeled temperatures and emission measures.

\begin{tabular}{|c|c|c|c|c|c|c|c|c|c|}
\hline Name & RA (J2000) & DEC (J2000) & $E M\left[c m^{-6} p c\right]$ & error EM & $\eta$ & error $\eta$ & $\tau_{5 \mathrm{GHz}}$ & $T_{b}^{\text {spec }}$ & $T_{b}^{\text {diam }}$ \\
\hline NGC 40 & 001301.010 & +723119.09 & $3.476 \mathrm{E}+5$ & $6.943 \mathrm{E}+4$ & 0.7283 & 0.0563 & 0.00338 & 37.158 & 18.260 \\
\hline Ни 1-1 & 002815.435 & +555754.48 & $4.206 \mathrm{E}+5$ & $3.375 \mathrm{E}+5$ & 0.0000 & & 0.00360 & 43.508 & 45.537 \\
\hline NGC 246 & 004703.338 & -115218.94 & $2.174 \mathrm{E}+3$ & $2.008 \mathrm{E}+5$ & 0.0000 & & 0.00001 & 0.205 & 0.214 \\
\hline NGC $650-51$ & 014219.69 & +513431.7 & $2.315 \mathrm{E}+5$ & $5.031 \mathrm{E}+4$ & 0.9907 & 0.0019 & 0.00234 & 24.995 & 0.485 \\
\hline IC 1747 & 015735.734 & +631918.33 & $4.005 \mathrm{E}+5$ & $1.284 \mathrm{E}+5$ & 0.5174 & 0.2177 & 0.00437 & 44.054 & 33.766 \\
\hline IC 289 & 031019.30 & +611901.0 & $8.199 \mathrm{E}+4$ & $9.264 \mathrm{E}+4$ & 0.6561 & 0.4832 & 0.00050 & 7.792 & 4.645 \\
\hline M 1-4 & 034143.439 & +521659.85 & $3.046 \mathrm{E}+6$ & $1.756 \mathrm{E}+6$ & 0.0000 & & 0.02419 & 305.881 & 320.152 \\
\hline IC 2003 & 035622.027 & +335229.27 & $4.048 \mathrm{E}+5$ & $1.118 \mathrm{E}+6$ & 0.0000 & & 0.00347 & 41.869 & 43.822 \\
\hline NGC 1501 & 040659.39 & +605514.4 & $3.304 \mathrm{E}+5$ & $5.757 \mathrm{E}+4$ & 0.9234 & 0.0126 & 0.00210 & 31.671 & 4.885 \\
\hline NGC 1514 & 040916.985 & +304633.47 & $7.418 \mathrm{E}+4$ & $3.716 \mathrm{E}+4$ & 0.9669 & 0.0163 & 0.00074 & 7.989 & 0.544 \\
\hline M 2-2 & 041315.043 & +565658.12 & $8.573 \mathrm{E}+5$ & $8.093 \mathrm{E}+5$ & 0.0000 & & 0.00726 & 88.263 & 92.380 \\
\hline NGC 1535 & 041415.765 & -124421.90 & $1.927 \mathrm{E}+5$ & $3.384 \mathrm{E}+4$ & 0.7032 & 0.0604 & 0.00173 & 20.182 & 10.678 \\
\hline IC 418 & 052728.203 & -124150.26 & $8.455 \mathrm{E}+6$ & $1.807 \mathrm{E}+6$ & 0.3525 & 0.2460 & 0.09743 & 900.472 & 825.399 \\
\hline NGC 2022 & 054206.20 & +090510.3 & $1.671 \mathrm{E}+5$ & $5.508 \mathrm{E}+4$ & 0.6989 & 0.1180 & 0.00108 & 16.099 & 8.620 \\
\hline M 1-5 & 054650.01 & +242202.8 & $7.838 \mathrm{E}+6$ & $1.145 \mathrm{E}+6$ & 0.3964 & 0.1215 & 0.09837 & 852.525 & 752.131 \\
\hline IC 2149 & 055623.901 & +4606 17.19 & $1.041 \mathrm{E}+6$ & $1.975 \mathrm{E}+5$ & 0.1948 & 0.4433 & 0.01107 & 113.344 & 114.128 \\
\hline IC 2165 & 062142.775 & -125913.96 & $4.652 \mathrm{E}+6$ & $1.113 \mathrm{E}+6$ & 0.7950 & 0.0501 & 0.03305 & 451.917 & 174.056 \\
\hline J 900 & 062557.237 & +174727.53 & $1.973 \mathrm{E}+6$ & $8.423 \mathrm{E}+5$ & 0.6452 & 0.1800 & 0.01690 & 202.745 & 123.857 \\
\hline M 1-6 & 063545.126 & -00 0537.36 & $1.108 \mathrm{E}+7$ & $9.299 \mathrm{E}+5$ & 0.6784 & 0.0240 & 0.11939 & 1147.872 & 648.450 \\
\hline NGC 2371 & 072534.68 & +292926.4 & $1.374 \mathrm{E}+5$ & $4.887 \mathrm{E}+4$ & 0.9216 & 0.0285 & 0.00096 & 13.496 & 2.127 \\
\hline NGC 2392 & 072910.765 & +205442.48 & $2.913 \mathrm{E}+5$ & $1.030 \mathrm{E}+5$ & 0.8226 & 0.0678 & 0.00174 & 27.486 & 9.301 \\
\hline NGC 2438 & 074150.51 & -144407.7 & $1.667 \mathrm{E}+6$ & $2.008 \mathrm{E}+6$ & 0.9975 & 0.0028 & 0.01643 & 177.596 & 0.926 \\
\hline NGC 2440 & 074154.91 & -181229.7 & $5.303 \mathrm{E}+5$ & $2.003 \mathrm{E}+5$ & 0.8018 & 0.0829 & 0.00373 & 52.150 & 19.491 \\
\hline Hen 2-9 & 082827.924 & -392339.92 & $4.864 \mathrm{E}+6$ & $4.600 \mathrm{E}+5$ & 0.4135 & 0.0560 & 0.05240 & 520.713 & 451.809 \\
\hline NGC 2610 & 083323.40 & -160857.5 & $1.042 \mathrm{E}+6$ & $2.562 \mathrm{E}+5$ & 0.9955 & 0.0010 & 0.00530 & 94.097 & 0.885 \\
\hline Hen 2-11 & 083708.10 & -39 2507.0 & $1.720 \mathrm{E}+5$ & $2.866 \mathrm{E}+4$ & 0.9189 & 0.0135 & 0.00185 & 18.883 & 3.074 \\
\hline Hen 2-15 & 085330.91 & -400342.3 & $2.074 \mathrm{E}+5$ & $1.167 \mathrm{E}+5$ & 0.7399 & 0.1666 & 0.00152 & 20.618 & 9.767 \\
\hline NGC 2867 & 092125.38 & -581840.9 & $1.396 \mathrm{E}+6$ & $2.150 \mathrm{E}+5$ & 0.5991 & 0.0750 & 0.01265 & 145.869 & 97.873 \\
\hline IC 2501 & 093847.146 & -600530.52 & $5.510 \mathrm{E}+6$ & $6.918 \mathrm{E}+5$ & 0.7746 & 0.0284 & 0.06262 & 594.869 & 249.031 \\
\hline NGC 3132 & 100701.764 & -402611.12 & $2.046 \mathrm{E}+5$ & $8.553 \mathrm{E}+4$ & 0.9436 & 0.0232 & 0.00226 & 22.607 & 2.595 \\
\hline Hen $2-47$ & 102309.14 & -603242.3 & $6.968 \mathrm{E}+6$ & $5.956 \mathrm{E}+5$ & 0.5406 & 0.0459 & 0.07522 & 739.082 & 547.105 \\
\hline NGC 3242 & 102446.107 & -183832.64 & $5.172 \mathrm{E}+5$ & $5.306 \mathrm{E}+4$ & 0.6945 & 0.0250 & 0.00486 & 54.735 & 29.656 \\
\hline IC 2621 & 110019.99 & -651457.8 & $2.760 \mathrm{E}+7$ & $1.244 \mathrm{E}+6$ & 0.7107 & 0.0143 & 0.21028 & 2503.267 & 1296.595 \\
\hline NGC 3587 & 111447.701 & +550108.72 & $1.550 \mathrm{E}+3$ & $8.467 \mathrm{E}+4$ & 0.0000 & & 0.00002 & 0.166 & 0.174 \\
\hline NGC 3918 & 115017.77 & -571056.4 & $3.916 \mathrm{E}+6$ & $4.138 \mathrm{E}+5$ & 0.7457 & 0.0292 & 0.03210 & 394.906 & 183.515 \\
\hline NGC 4361 & 122430.76 & -18 4705.4 & $1.884 \mathrm{E}+6$ & $4.679 \mathrm{E}+5$ & 0.9974 & 0.0005 & 0.00835 & 163.881 & 0.876 \\
\hline IC 3568 & 123306.871 & +823348.95 & $6.186 \mathrm{E}+5$ & $1.398 \mathrm{E}+5$ & 0.8415 & 0.0380 & 0.00649 & 67.305 & 20.559 \\
\hline NGC 5307 & 135103.322 & -511220.77 & $3.122 \mathrm{E}+5$ & $1.570 \mathrm{E}+5$ & 0.5694 & 0.2821 & 0.00251 & 31.779 & 22.478 \\
\hline NGC 5315 & 135357.00 & -663050.7 & $8.513 \mathrm{E}+6$ & $7.811 \mathrm{E}+5$ & 0.8083 & 0.0172 & 0.10529 & 919.434 & 333.545 \\
\hline Hen 2-113 & 145953.476 & -541807.42 & $5.718 \mathrm{E}+7$ & $2.292 \mathrm{E}+7$ & 0.0000 & & 0.61588 & 4690.332 & 4910.109 \\
\hline Hen 2-142 & 155957.63 & -555533.0 & $9.975 \mathrm{E}+6$ & $1.195 \mathrm{E}+6$ & 0.7862 & 0.0258 & 0.10745 & 1039.136 & 415.443 \\
\hline IC 4593 & 161144.544 & +120417.06 & $1.578 \mathrm{E}+6$ & $5.534 \mathrm{E}+5$ & 0.9192 & 0.0255 & 0.02040 & 179.739 & 29.183 \\
\hline NGC 6072 & 161258.40 & -361347.0 & $6.337 \mathrm{E}+4$ & $3.877 \mathrm{E}+4$ & 0.8552 & 0.0945 & 0.00059 & 6.701 & 1.884 \\
\hline NGC 6153 & 163130.626 & -401512.31 & $1.603 \mathrm{E}+6$ & $3.786 \mathrm{E}+5$ & 0.7782 & 0.0563 & 0.01541 & 169.767 & 70.093 \\
\hline NGC 6210 & 164429.491 & +234759.68 & $9.176 \mathrm{E}+5$ & $6.085 \mathrm{E}+5$ & 0.0000 & & 0.01057 & 102.019 & 106.778 \\
\hline IC 4634 & 170133.57 & -214933.3 & $1.171 \mathrm{E}+6$ & $1.229 \mathrm{E}+5$ & 0.7378 & 0.0286 & 0.01331 & 129.607 & 61.816 \\
\hline NGC 6302 & 171344.339 & -370610.95 & $9.779 \mathrm{E}+6$ & $2.545 \mathrm{E}+6$ & 0.9678 & 0.0073 & 0.05513 & 884.954 & 58.724 \\
\hline NGC 6309 & 171404.299 & -125435.74 & $3.615 \mathrm{E}+5$ & $8.531 \mathrm{E}+4$ & 0.4552 & 0.1719 & 0.00339 & 38.289 & 31.772 \\
\hline H 1-13 & 172827.503 & -350731.58 & $3.514 \mathrm{E}+6$ & $1.464 \mathrm{E}+6$ & 0.5394 & 0.2006 & 0.03785 & 378.898 & 281.174 \\
\hline NGC 6369 & 172920.45 & -234534.8 & $2.369 \mathrm{E}+6$ & $5.004 \mathrm{E}+5$ & 0.5832 & 0.1157 & 0.01942 & 240.382 & 166.032 \\
\hline $\mathrm{Hb} 4$ & 174152.80 & -244208.7 & $3.319 \mathrm{E}+6$ & $4.091 \mathrm{E}+5$ & 0.7387 & 0.0346 & 0.01942 & 353.915 & 168.292 \\
\hline $\mathrm{Hb} 5$ & 174756.20 & -295939.6 & $2.668 \mathrm{E}+7$ & $6.677 \mathrm{E}+6$ & 0.9933 & 0.0007 & 0.03396 & 2404.431 & 33.666 \\
\hline NGC 6445 & 174915.21 & -200034.5 & $1.858 \mathrm{E}+6$ & $6.527 \mathrm{E}+5$ & 0.9934 & 0.0022 & 0.01665 & 193.196 & 2.675 \\
\hline NGC 6543 & 175833.423 & +663759.52 & $1.112 \mathrm{E}+6$ & $7.615 \mathrm{E}+4$ & 0.5645 & 0.0379 & 0.01630 & 130.998 & 93.416 \\
\hline NGC 6537 & 180513.104 & -195034.88 & $1.126 \mathrm{E}+7$ & $9.717 \mathrm{E}+5$ & 0.8038 & 0.0164 & 0.06789 & 1030.472 & 381.725 \\
\hline M 1-40 & 180825.989 & -221652.93 & $3.543 \mathrm{E}+6$ & $2.567 \mathrm{E}+6$ & 0.7090 & 0.2182 & 0.02936 & 358.767 & 186.746 \\
\hline M 1-41 & 180930.10 & -241226.0 & $3.087 \mathrm{E}+6$ & $2.685 \mathrm{E}+6$ & 0.9940 & 0.0042 & 0.03042 & 326.550 & 4.080 \\
\hline NGC 6572 & 181206.365 & +065113.01 & $1.532 \mathrm{E}+7$ & $1.172 \mathrm{E}+6$ & 0.8508 & 0.0111 & 0.16286 & 1547.986 & 447.504 \\
\hline
\end{tabular}




\begin{tabular}{|c|c|c|c|c|c|c|c|c|c|}
\hline SwSt 1 & 181612.268 & -305208.01 & $6.488 \mathrm{E}+7$ & $6.079 \mathrm{E}+6$ & 0.9599 & 0.0029 & 0.69886 & 5129.055 & 422.482 \\
\hline NGC 6578 & 181616.517 & -202702.67 & $9.625 \mathrm{E}+5$ & $8.108 \mathrm{E}+5$ & 0.5474 & 0.5162 & 0.01037 & 105.203 & 77.117 \\
\hline Cn 3-1 & 181734.113 & +100903.46 & $7.673 \mathrm{E}+6$ & $2.850 \mathrm{E}+6$ & 0.8544 & 0.0512 & 0.03022 & 639.986 & 180.845 \\
\hline NGC 6629 & 182542.458 & $-2312 \quad 10.23$ & $1.613 \mathrm{E}+6$ & $7.764 \mathrm{E}+5$ & 0.7826 & 0.1069 & 0.02117 & 184.324 & 74.756 \\
\hline M 2-43 & 182640.05 & -024257.3 & $1.146 \mathrm{E}+8$ & $1.381 \mathrm{E}+7$ & 0.5506 & 0.0539 & 1.23460 & 7232.283 & 5277.104 \\
\hline M 1-51 & 183329.05 & -110726.5 & $3.911 \mathrm{E}+6$ & $.599 \mathrm{E}+6$ & 0.7893 & 0.0858 & 0.04212 & 420.741 & 166.031 \\
\hline M 1-59 & 184320.20 & -09 0449.1 & $6.214 \mathrm{E}+6$ & $.119 \mathrm{E}+6$ & 0.8287 & 0.0263 & 0.06693 & 660.372 & 216.476 \\
\hline M 1-61 & 184555.12 & -142737.9 & $3.085 \mathrm{E}+7$ & $2.752 \mathrm{E}+6$ & 0.3319 & 0.0728 & 0.33230 & 2883.860 & 2686.219 \\
\hline Hu 2-1 & 184947.567 & +205039.45 & $.942 \mathrm{E}+7$ & $1.565 \mathrm{E}+6$ & 0.9028 & 0.0051 & 0.22686 & 1948.460 & 377.028 \\
\hline NGC 6720 & 185335.079 & +330145.03 & $1.438 \mathrm{E}+5$ & $.327 \mathrm{E}+4$ & 0.8529 & 0.0474 & 0.00138 & & 4.375 \\
\hline K $3-17$ & 185618.164 & +070725.88 & $3.817 \mathrm{E}+6$ & $9.507 \mathrm{E}+5$ & 0.8458 & 0379 & 0.04111 & 410.849 & 122.363 \\
\hline M 1-66 & 185826.24 & -010345.6 & $7.136 \mathrm{E}+6$ & $2.707 \mathrm{E}+6$ & 0.5779 & 0.1432 & 0.07687 & & 526.094 \\
\hline NGC 6741 & 190237.10 & -002656.7 & $34 \mathrm{E}+7$ & $5 \mathrm{E}+6$ & 0.8794 & 0.0179 & 8388 & 822 & 240.583 \\
\hline Abell 53 & 190645.910 & +062352.47 & $8 \mathrm{E}+7$ & $3.379 \mathrm{E}+6$ & 986 & & 5997 & 1788.741 & 5.234 \\
\hline Hen $2-430$ & 191404.13 & +173133.1 & $1 \mathrm{E}+7$ & $7.609 \mathrm{E}+5$ & 0.9084 & 0.0024 & & 1597.668 & 292.355 \\
\hline NGC 6781 & 191828.085 & +063219.29 & $1.358 \mathrm{E}+4$ & $4.336 \mathrm{E}+5$ & 0.0000 & & 0.00015 & 1.502 & 1.572 \\
\hline NGC 6790 & 192256.966 & +013046.46 & $6.330 \mathrm{E}+7$ & $3.213 \mathrm{E}+6$ & 0.8698 & 0.0055 & 0.50259 & 5056.511 & 1288.885 \\
\hline Vy 2-2 & 192422.223 & +095356.29 & $.476 \mathrm{E}+8$ & $8.626 \mathrm{E}+7$ & 0.9802 & 0.0021 & 7563 & .46 & 419.323 \\
\hline K 3-35 & 192744.02 & +213003.4 & $\mathrm{E}+7$ & $E+6$ & 629 & & & .072 & 196.957 \\
\hline PB 10 & 192814.487 & +12 1937.11 & $3 \mathrm{E}+6$ & $5.816 \mathrm{E}+5$ & 3975 & 0.0336 & & & \\
\hline NGC 6803 & 193116.47 & +100321.7 & $3 \mathrm{E}+6$ & $1.006 \mathrm{E}+6$ & 0.8254 & 0.0170 & & & 280.474 \\
\hline NGC 6804 & 193135.14 & +09 1331.4 & $\mathrm{E}+6$ & $\mathrm{E}+6$ & & & & & 3.138 \\
\hline $\mathrm{BD}+303639$ & 193445.233 & +303058.94 & +7 & $\mathrm{E}+6$ & & & & 857 & 1332.475 \\
\hline M 1-71 & 26.92 & +194224.1 & $\mathrm{E}+7$ & $1 \mathrm{E}+5$ & & 0.0066 & & 664 & \\
\hline M 1-73 & 194109.29 & +145658.8 & $\mathrm{E}+5$ & $9.091 \mathrm{E}+5$ & 0.1400 & & & & 60.604 \\
\hline NGC 6818 & 194358.022 & -140913.44 & $\mathrm{E}+5$ & $5.174 \mathrm{E}+4$ & & 0.0841 & 324 & & 34.278 \\
\hline NGC 6826 & 194448.150 & +503130 & & $\mathrm{E}+5$ & & & & & \\
\hline Hen $2-447$ & 22.16 & +212003.9 & +7 & $\mathrm{E}+6$ & & 360 & & 197 & 1161.339 \\
\hline NGC 6842 & 195502.1 & +291722 & $E+5$ & $\mathrm{E}+5$ & & & & & 0.953 \\
\hline Hen 1-4 & 195918.014 & +315439.14 & $\mathrm{E}+6$ & $1.249 \mathrm{E}+7$ & 0.9981 & 0.0012 & 743 & 1018.633 & 4.074 \\
\hline K 3-52 & 200311.44 & +303234.1 & $\mathrm{E}+7$ & $3.695 \mathrm{E}+6$ & & 0.0108 & & 3276.799 & 930. \\
\hline M 1-75 & 200444.1 & +312728 & +5 & $\mathrm{E}+4$ & & 0.0019 & 367 & & 590 \\
\hline K 3-55 & 200656.250 & +321636.80 & $\mathrm{E}+6$ & $\mathrm{E}+5$ & & & & & \\
\hline NGC 6884 & 201023.66 & +462739.8 & $2 \mathrm{E}+6$ & $5.046 \mathrm{E}+5$ & 0.6440 & 0.0526 & 893 & & 240.900 \\
\hline NGC 6881 & 52.45 & +372442.4 & $8.812 \mathrm{E}+6$ & $2.081 \mathrm{E}+6$ & & 0.0130 & & & 126.555 \\
\hline NGC 68 & 42.83 & +195922.6 & +6 & $\mathrm{E}+6$ & & 0.0783 & & & 151.363 \\
\hline K 3-57 & 201247.725 & +342032.79 & $\mathrm{E}+5$ & $8 \mathrm{E}+6$ & 0.0000 & & 0767 & & 81.586 \\
\hline Hen 2-459 & 201357.89 & +293355.9 & $4 \mathrm{E}+7$ & $7.524 \mathrm{E}+6$ & & & & 4153.345 & 876.039 \\
\hline NGC 6891 & 201508.838 & +124215.63 & $2.839 \mathrm{E}+6$ & $8.101 \mathrm{E}+5$ & 0.9168 & 0.0213 & 0.03058 & 307.163 & 51.258 \\
\hline NGC 6894 & 23.965 & +303353.17 & $\mathrm{E}+5$ & $2.284 \mathrm{E}+5$ & & & & & \\
\hline IC 4997 & 08.742 & +164353.71 & $\mathrm{E}+7$ & $\mathrm{E}+6$ & 0.7952 & 0.0417 & 786 & 3219.897 & 1238.914 \\
\hline M 3-35 & 202103.77 & +322924.0 & $96 \mathrm{E}+7$ & $3.791 \mathrm{E}+6$ & 0.9272 & 0.0044 & 0.49506 & 3982.741 & 585.173 \\
\hline NGC 6905 & 202222.991 & +200616.25 & $2.252 \mathrm{E}+6$ & $1.514 \mathrm{E}+6$ & 0.9933 & 0.0041 & 0.01928 & 231.096 & 3.226 \\
\hline NGC 7008 & 210032.7 & +543239 & $1.096 \mathrm{E}+5$ & $2.465 \mathrm{E}+4$ & 0.9138 & & & & \\
\hline NGC 7009 & 210410.877 & -112148.26 & & $5.440 \mathrm{E}+4$ & & & & & \\
\hline NGC 7026 & $2106 \quad 18.237$ & +475107.15 & $9.590 \mathrm{E}+5$ & $7.862 \mathrm{E}+4$ & 0.8750 & 0.0075 & 0.01204 & 108.877 & 26.709 \\
\hline NGC 7027 & 210701.8 & +421410 & $5.450 \mathrm{E}+7$ & $9.574 \mathrm{E}+6$ & 0.6965 & 0.0630 & 0.45157 & 4505.795 & 2428.366 \\
\hline NGC 7048 & 211415.25 & +461716.1 & $1.885 \mathrm{E}+5$ & $1.909 \mathrm{E}+5$ & 0.9820 & 0.0174 & 0.00153 & 19.248 & 0.719 \\
\hline K 3-62 & 213150.18 & +523351.6 & $9.779 \mathrm{E}+6$ & $1.930 \mathrm{E}+6$ & & & & 1019.792 & 478.198 \\
\hline IC 5117 & 213230.97 & +443547.5 & $6.225 \mathrm{E}+7$ & $2.563 \mathrm{E}+6$ & 0.7291 & 0.0092 & 0.56422 & 5001.907 & 2452.423 \\
\hline $\mathrm{Hu} 1-2$ & 213308.328 & +393809.72 & $3.021 \mathrm{E}+6$ & $3.404 \mathrm{E}+6$ & 0.0000 & & 0.03529 & 332.913 & 348.447 \\
\hline Bl 2-1 & 222016.63 & +581416.6 & $1.637 \mathrm{E}+7$ & $2.118 \mathrm{E}+6$ & 0.4362 & 0.0782 & 0.17631 & 1648.746 & 1397.387 \\
\hline IC 5217 & 222355.73 & +505800.5 & $1.504 \mathrm{E}+6$ & $9.728 \mathrm{E}+5$ & 0.7386 & 0.1812 & 0.01363 & 157.056 & 74.700 \\
\hline Me 2-2 & 223143.683 & +474803.91 & $2.123 \mathrm{E}+7$ & $1.951 \mathrm{E}+6$ & 0.7895 & 0.0127 & 0.21177 & 2061.190 & 812.550 \\
\hline NGC 7354 & 224019.83 & +611708.7 & $7.675 \mathrm{E}+5$ & $1.342 \mathrm{E}+5$ & 0.7503 & 0.0446 & 0.00650 & 79.049 & 36.155 \\
\hline NGC 7662 & 232553.6 & +423206 & $8.681 \mathrm{E}+5$ & $1.240 \mathrm{E}+5$ & 0.6821 & 0.0508 & 0.00689 & 87.918 & 49.206 \\
\hline
\end{tabular}



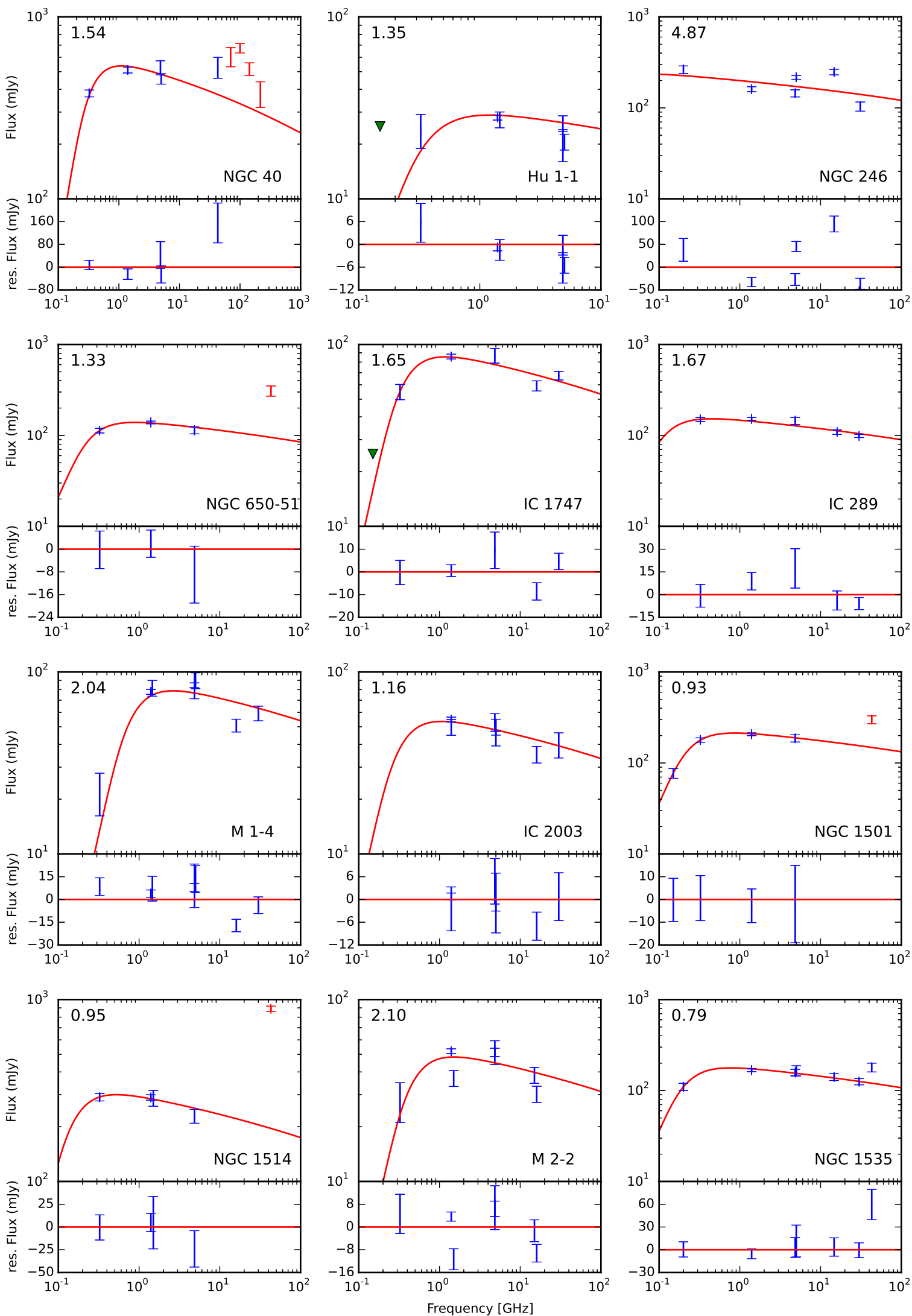

Figure B1. Radio spectra and best model fits for PNe radio spectra. Triangles set an upper limit for the TGSS survey, if it is lower than the next data point. Red errorbars mark data not used in the fit. 

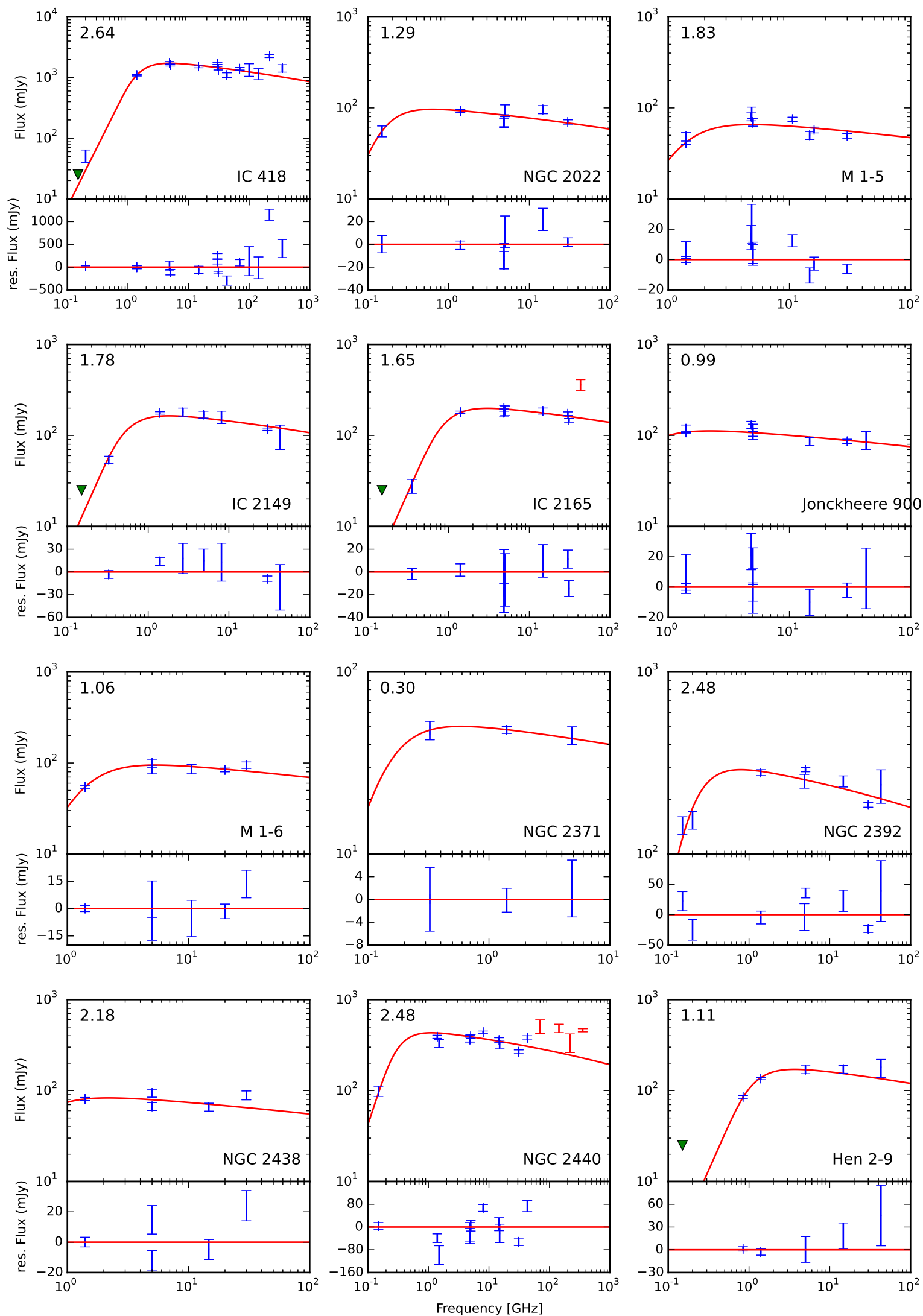

Figure B2. Radio spectra and best model fits for PNe radio spectra. Triangles set an upper limit for the TGSS survey, if it is lower than the next data point. Red errorbars mark data not used in the fit. 

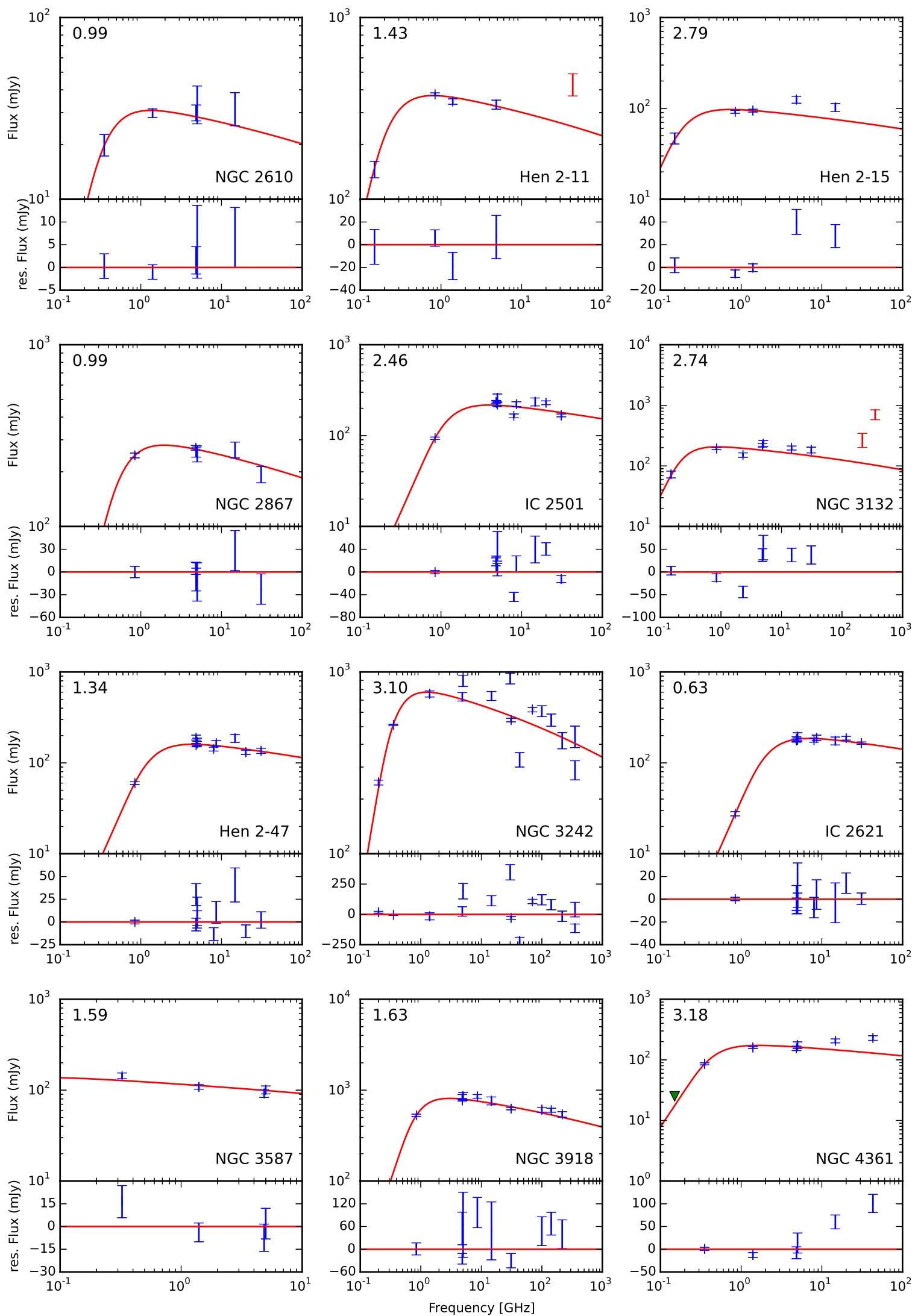

Figure B3. Radio spectra and best model fits for PNe radio spectra. Triangles set an upper limit for the TGSS survey, if it is lower than the next data point. Red errorbars mark data not used in the fit. 

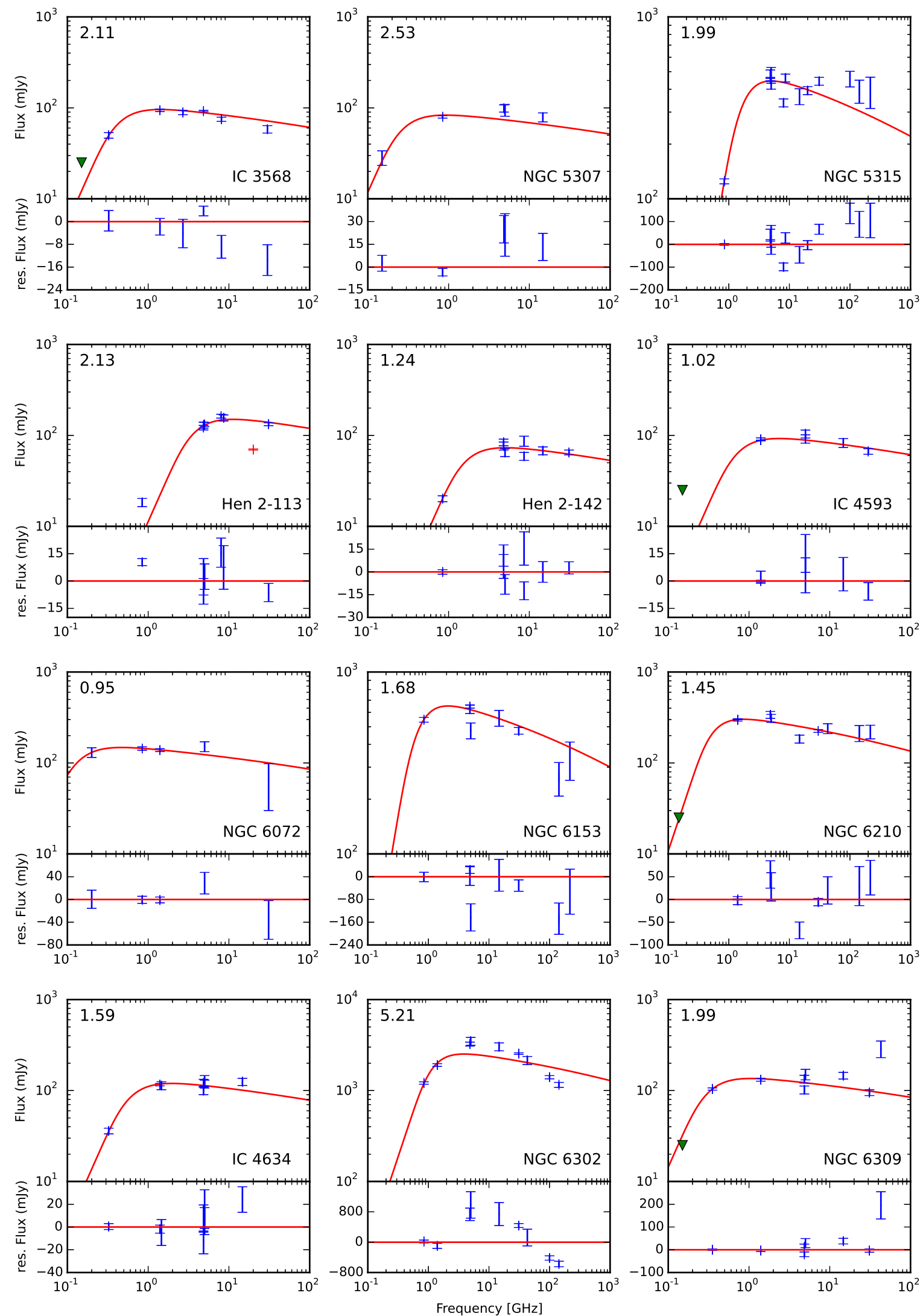

Figure B4. Radio spectra and best model fits for PNe radio spectra. Triangles set an upper limit for the TGSS survey, if it is lower than the next data point. Red errorbars mark data not used in the fit. 

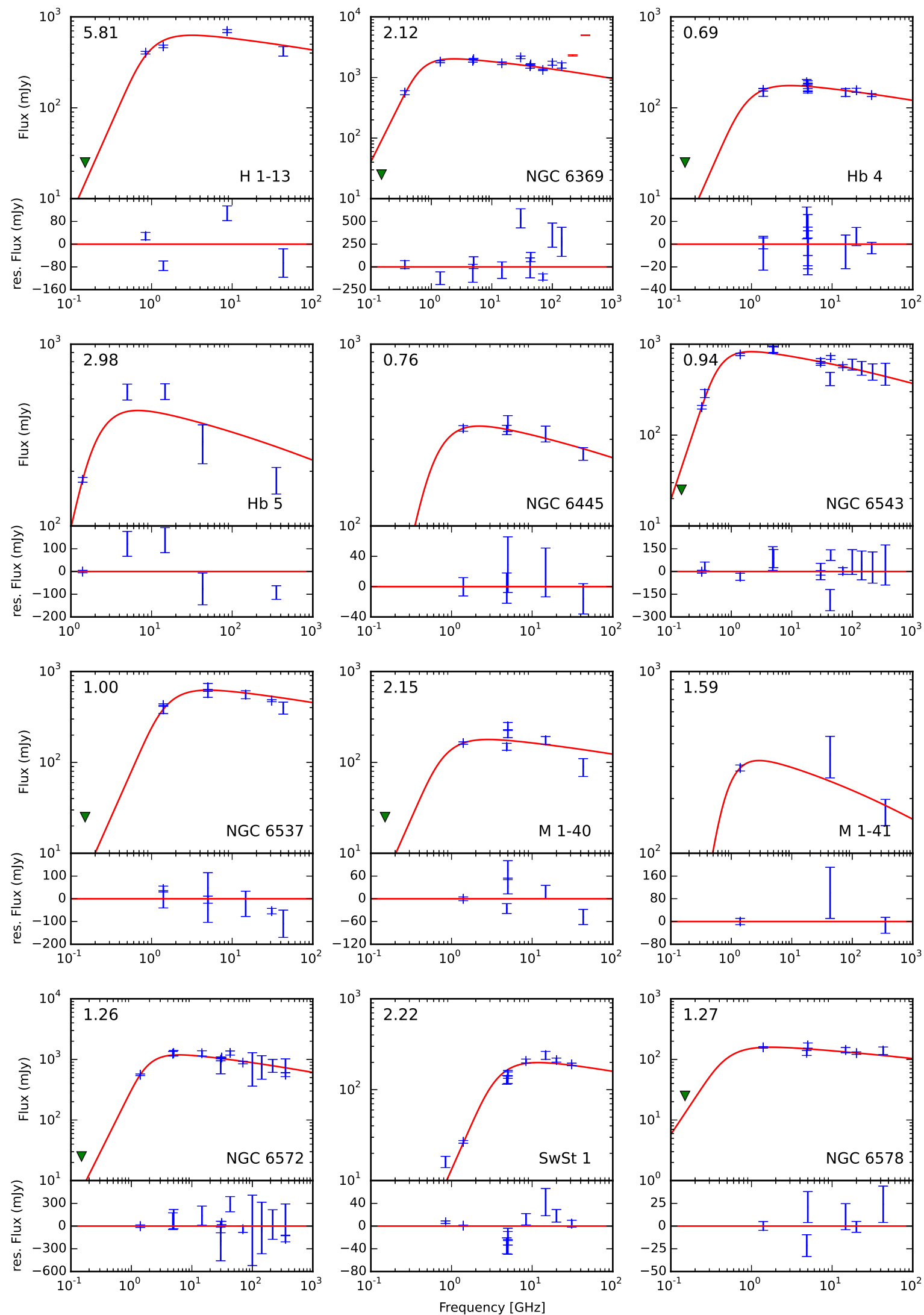

Figure B5. Radio spectra and best model fits for PNe radio spectra. Triangles set an upper limit for the TGSS survey, if it is lower than the next data point. Red errorbars mark data not used in the fit. 

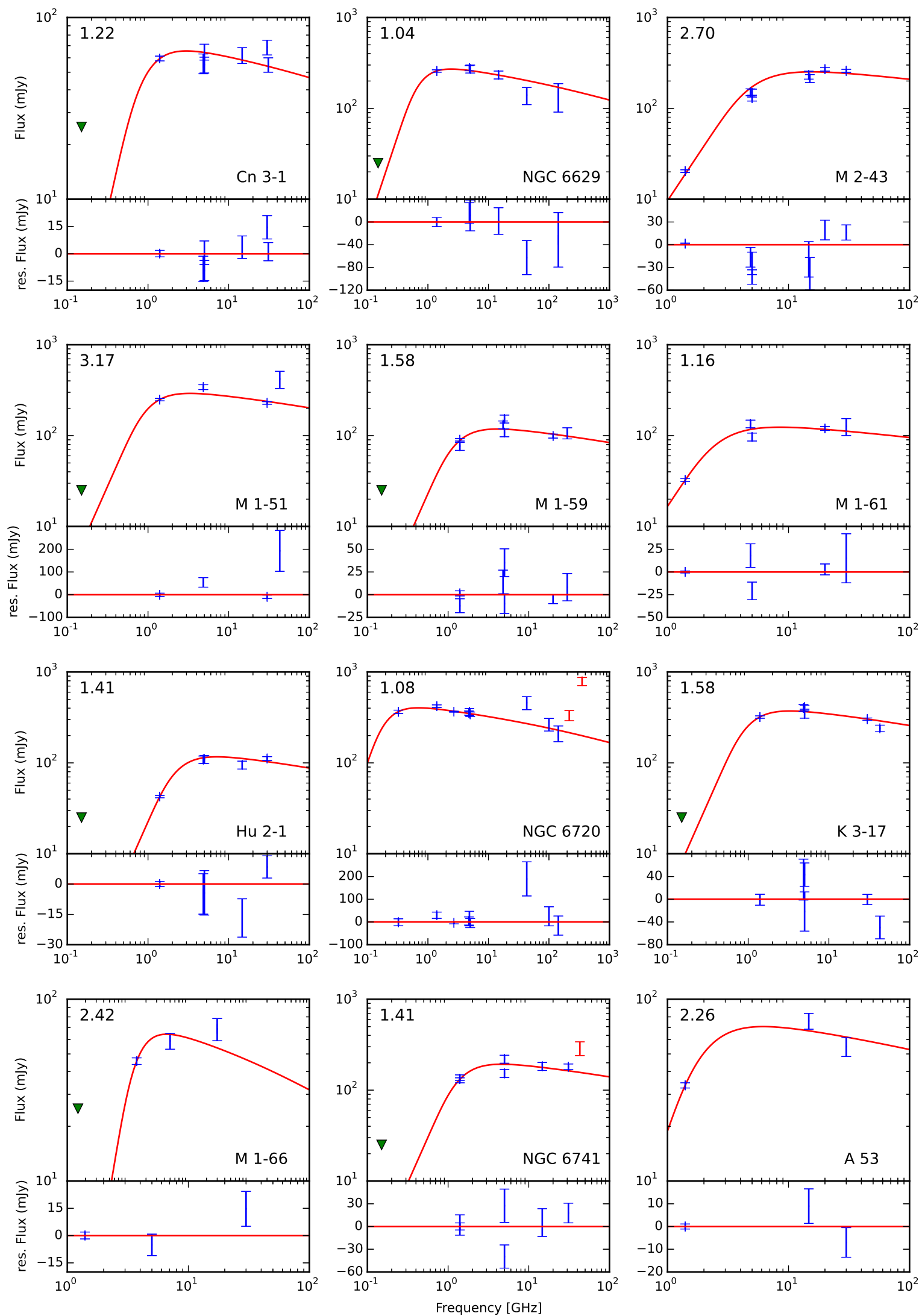

Figure B6. Radio spectra and best model fits for PNe radio spectra. Triangles set an upper limit for the TGSS survey, if it is lower than the next data point. Red errorbars mark data not used in the fit. 

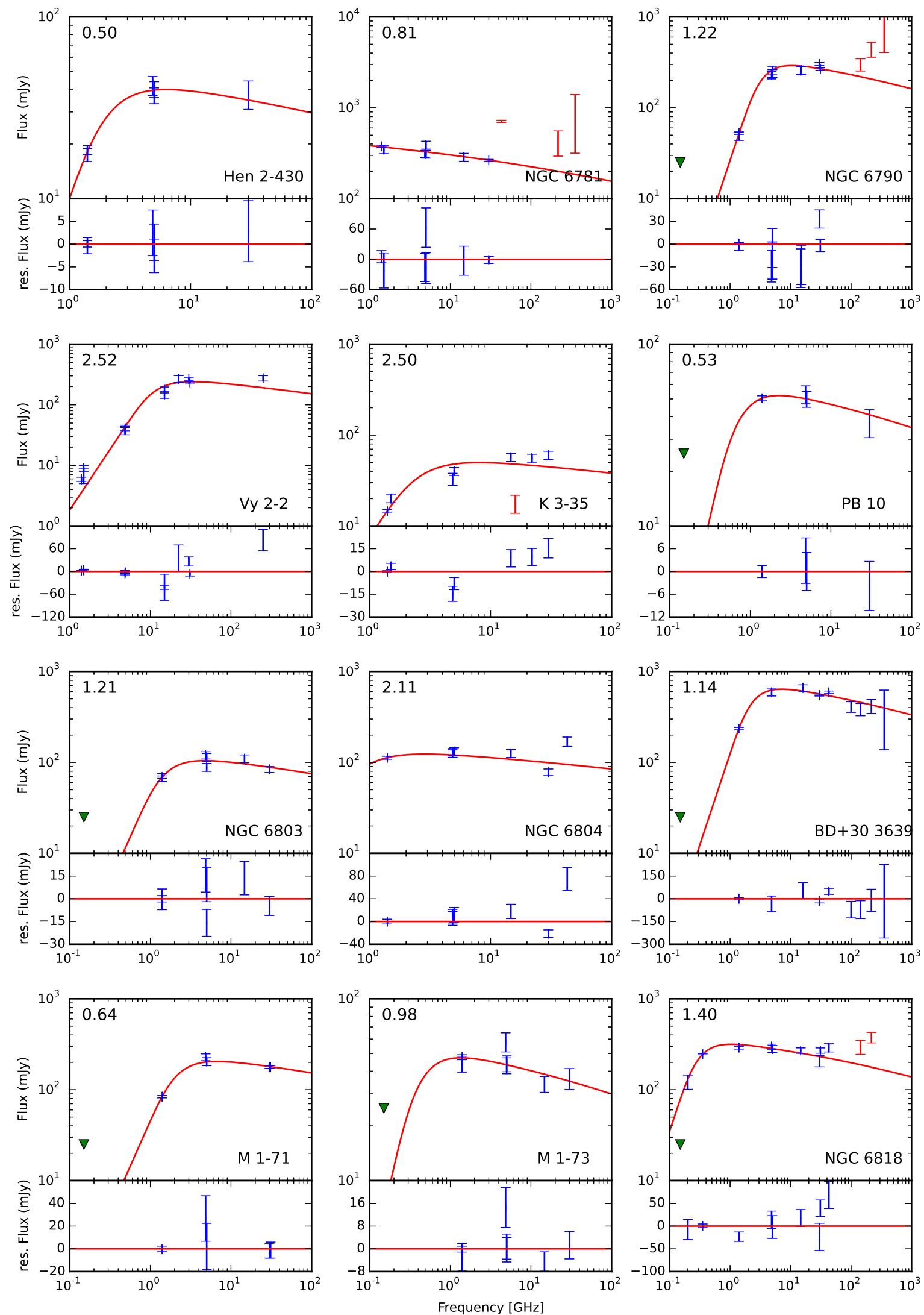

Figure B7. Radio spectra and best model fits for PNe radio spectra. Triangles set an upper limit for the TGSS survey, if it is lower than the next data point. Red errorbars mark data not used in the fit. 

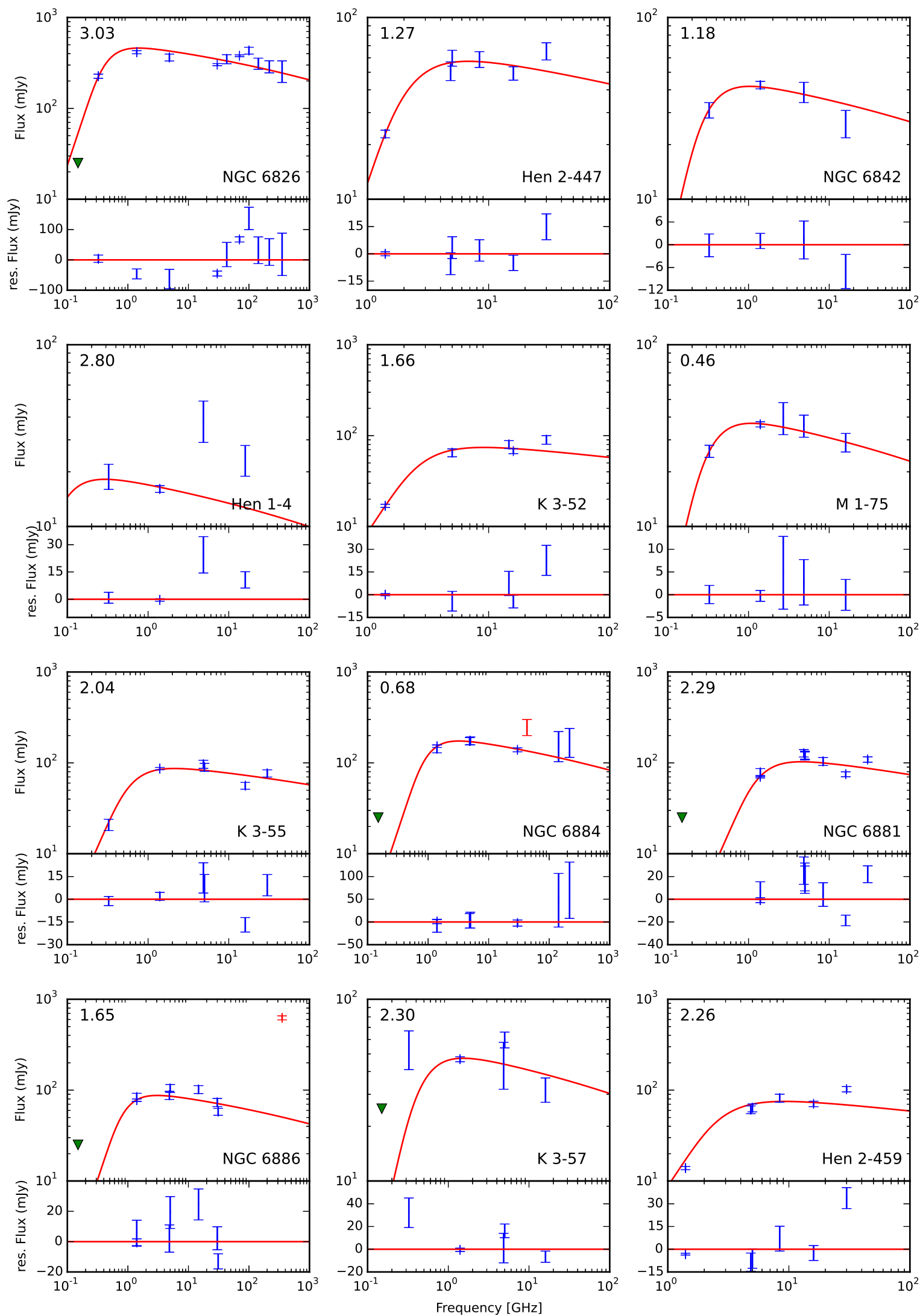

Figure B8. Radio spectra and best model fits for PNe radio spectra. Triangles set an upper limit for the TGSS survey, if it is lower than the next data point. Red errorbars mark data not used in the fit. 

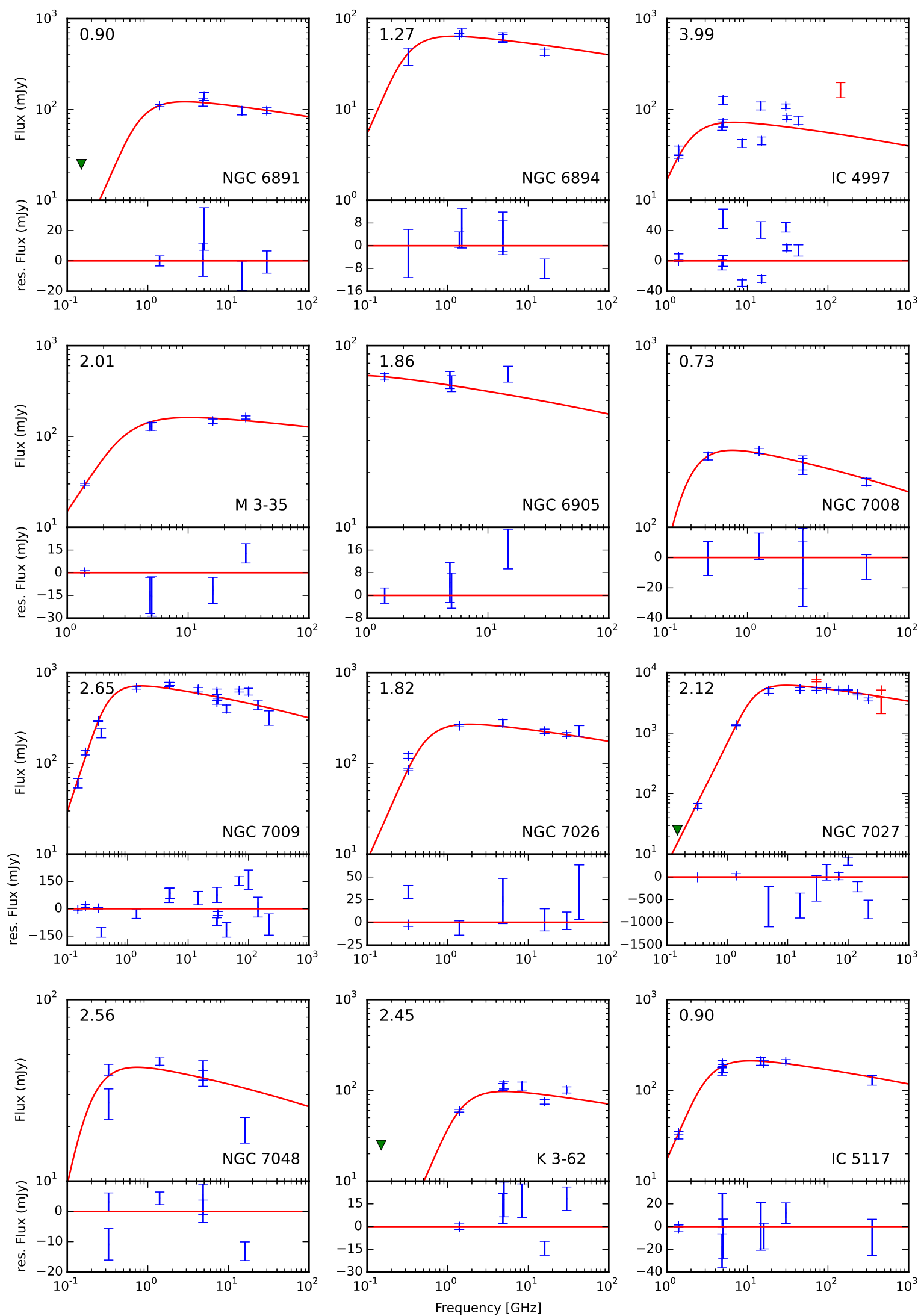

Figure B9. Radio spectra and best model fits for PNe radio spectra. Triangles set an upper limit for the TGSS survey, if it is lower than the next data point. Red errorbars mark data not used in the fit. 

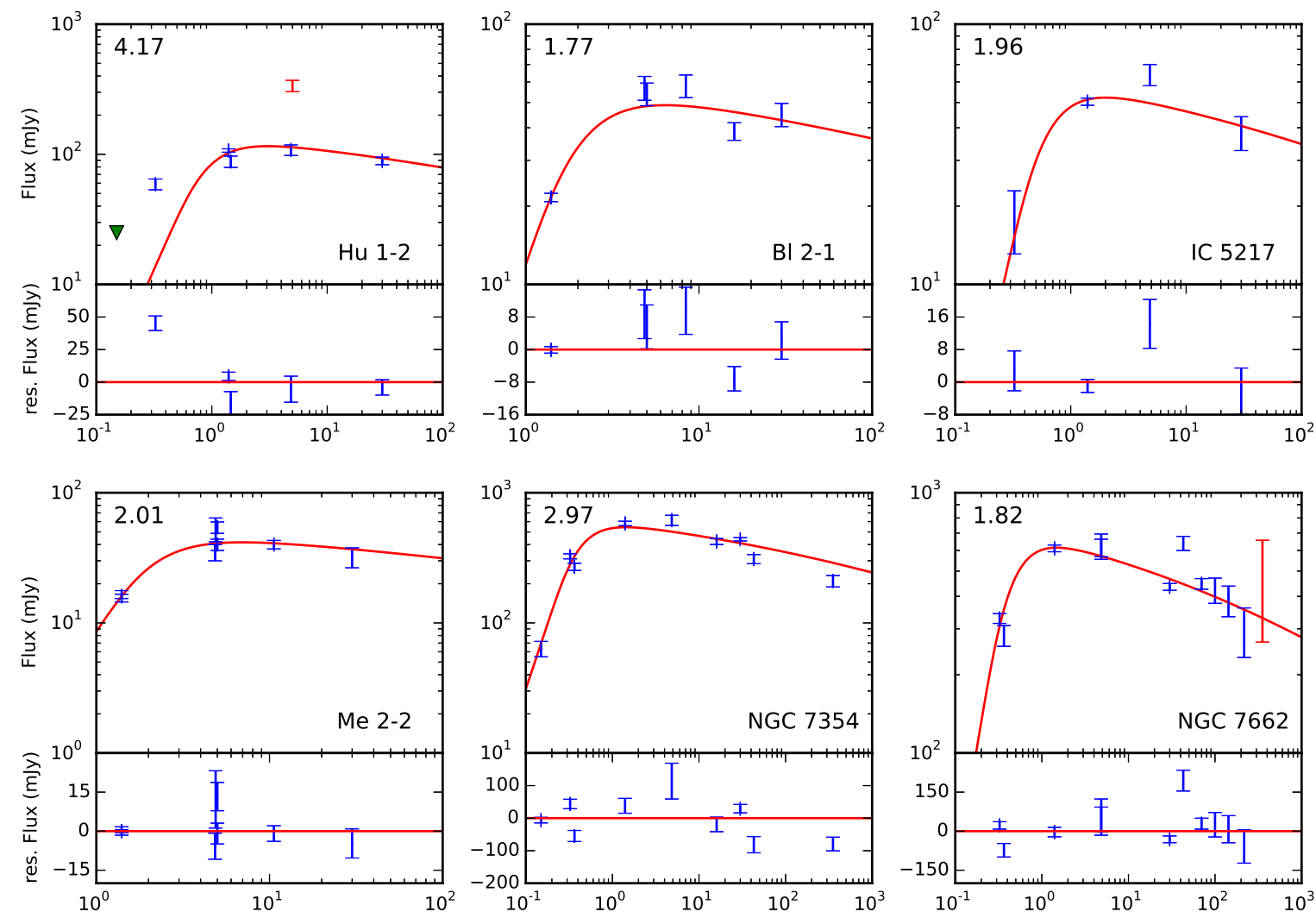

Figure B10. Radio spectra and best model fits for PNe radio spectra. Triangles set an upper limit for the TGSS survey, if it is lower than the next data point. Red errorbars mark data not used in the fit. 
22 M. Hajduk et al.

APPENDIX B: SPECTRA OF INDIVIDUAL PNE

This paper has been typeset from a $\mathrm{TEX}_{\mathrm{E}} / \mathrm{LATEX}$ file prepared by the author. 